\title{
NONTRIVIAL SOLUTIONS FOR A MIXED BOUNDARY PROBLEM FOR SCHRÖDINGER EQUATIONS WITH AN EXTERNAL MAGNETIC FIELD
}

\author{
Claudianor O. Alves - Rodrigo C.M. Nemer \\ SÉrgio H.M. SoAres
}

(Submitted by Jean Mawhin)

\begin{abstract}
We study the existence of solutions for a class of nonlinear Schrödinger equations involving a magnetic field with mixed DirichletNeumann boundary conditions. We use Lusternik-Shnirelman category and the Morse theory to estimate the number of nontrivial solutions in terms of the topology of the part of the boundary where the Neumann condition is prescribed.
\end{abstract}

\section{Introduction}

A major role in quantum physics is played by the nonlinear Schrödinger equation

$$
i h \frac{\partial \Psi}{\partial t}=\left(\frac{h}{i} \nabla-A(x)\right)^{2} \Psi+U(x) \Psi-f\left(|\Psi|^{2}\right) \Psi, \quad x \in \Omega,
$$

2010 Mathematics Subject Classification. Primary: 35A15, 14E20; Secondary: 35H30, $35 \mathrm{Q} 55$.

Key words and phrases. Nonlinear Schrödinger equation, variational methods, LusternikSchnirelman category, Morse theory.

C.O. Alves was partially supported by $\mathrm{CNPq}$

R.C.M. Nemer was supported by FAPESP.

Corresponding author. S.H.M. Soares was partially supported by CNPq. 
where $\Omega$ is a bounded smooth domain in $\mathbb{R}^{N}, N \geq 3, t \in \mathbb{R}, h$ is a positive constant, $i$ is the imaginary unit, $\Psi: \mathbb{R} \times \mathbb{R}^{N} \rightarrow \mathbb{C}$ is the wave function, $f$ is a nonlinear term, $U$ is the real electric potential, $A: \mathbb{R}^{N} \rightarrow \mathbb{R}^{N}$ denotes a magnetic potential and the Schrödinger operator is defined by

$$
\left(\frac{h}{i} \nabla-A(x)\right)^{2} \Psi=-h^{2} \Delta \Psi-\frac{2 h}{i} A \nabla \Psi+|A|^{2} \Psi-\frac{h}{i} \Psi \operatorname{div} A .
$$

We are interested in standing wave solutions, that is, solutions for (1.1) in the form $\Psi(t, x)=e^{-i E t / h} u(x)$, where $u$ satisfies

$$
\left(\frac{h}{i} \nabla-A(x)\right)^{2} u+V(x) u=f\left(|u|^{2}\right) u, \quad x \in \Omega,
$$

where $V(x)=U(x)-E$. Assuming that $V \equiv 1$, it follows immediately that $u$ is a solution of 2 if, and only if, the function $v(x)=u(h x)$ solves

$$
\left(\frac{1}{i} \nabla-A_{\lambda}(x)\right)^{2} v+v=f\left(|v|^{2}\right) v, \quad x \in \Omega_{\lambda},
$$

where $\lambda=h^{-1}, A_{\lambda}(x)=A\left(\lambda^{-1} x\right)$ and $\Omega_{\lambda} \doteq \lambda \Omega$. The case with no magnetic vector field, namely $A=0$, has been widely studied in the literature. We refer to [3], [4], [7], [8], [20], [23], [27], [33], [34], and references in these papers. Existence results for the magnetic case were established in [1], [2], [5], [12]-[18], [22], [24]-[26], [28], [30]-[32]. In [2], the authors have proved that if $f$ is a superlinear function with subcritical growth, then for large values of $\lambda>0$, the equation (1.3) with boundary Dirichlet condition has at least cat $\Omega_{\Omega_{\lambda}}\left(\Omega_{\lambda}\right)$ nontrivial weak solutions, where $\operatorname{cat}_{\Omega_{\lambda}}\left(\Omega_{\lambda}\right)$ denotes the the Lusternik-Schnirelman category of $\Omega_{\lambda}$ in $\Omega_{\lambda}$. In the seminal works [7], [8], Benci and Cerami used Lusternik-Schnirelman category and Morse theory to estimate the number of positive solutions of the problem

$$
-\varepsilon \Delta u+u=f(u), \quad \text { in } \Omega, \quad u=0, \quad \text { on } \partial \Omega,
$$

where $\Omega$ is a bounded domain. In [8], it is proved that for $\varepsilon$ sufficiently small the number of positive solutions is at least $\operatorname{cat}_{\Omega}(\Omega)$. In [7], the authors proved via Morse theory that the number of solutions depends on the topology of $\Omega$, actually on $\mathcal{P}_{t}(\Omega)$, the Poincaré polynomial of $\Omega$. In [11], Candela and Lazzo have considered this same equation with mixed Dirichlet-Neumann boundary conditions with $f(t)=|t|^{p-2} t$. It was proved that the number of positive solutions is influenced by the topology of the part $\Gamma_{1}$ of the boundary $\partial \Omega$ where the Neumann condition is assumed, more precisely, if $(N-1)$-dimensional Lebesgue measure in $\mathbb{R}^{N}$ is positive, then the respective problem has at least category of a set $\Gamma_{1}$, provided $\varepsilon$ is sufficiently small. 
Motivated by the results just described, a natural question is whether same kind of result holds for the mixed boundary problem with magnetic field

$$
\begin{cases}\left(-i \nabla-A_{\lambda}\right)^{2} u+u=f\left(|u|^{2}\right) u & \text { in } \Omega_{\lambda}, \\ u=0 & \text { on } \Gamma_{0 \lambda}, \\ \frac{\partial u}{\partial \nu}=0, & \text { on } \Gamma_{1 \lambda},\end{cases}
$$

where $\lambda$ is a positive real parameter, $\Omega_{\lambda}=\lambda \Omega$ is an expanding set, $\Omega \subset \mathbb{R}^{N}$ $(N \geq 3)$ is a bounded domain with smooth boundary $\partial \Omega=\overline{\Gamma_{0}} \cup \overline{\Gamma_{1}}$, where $\Gamma_{0}, \Gamma_{1}$ are smooth disjoint submanifolds with positive $(N-1)$-dimensional Lebesgue measure in $\mathbb{R}^{N}, \Gamma_{0 \lambda} \doteq \lambda \Gamma_{0}, \Gamma_{1 \lambda} \doteq \lambda \Gamma_{1}, A \in C\left(\Omega, \mathbb{R}^{N}\right)$ and $f \in C^{1}\left(\mathbb{R}^{+}\right)$satisfies:

$\left(\mathrm{f}_{1}\right) f(s)=o(1)$ and $f^{\prime}(s)=o(1 / s)$, as $s \rightarrow 0^{+}$.

$\left(\mathrm{f}_{2}\right)$ There exists $q \in\left(2,2^{*}\right)$ such that

$$
\lim _{s \rightarrow \infty} \frac{f(s)}{s^{(q-2) / 2}}=0 \quad \text { and } \quad \lim _{s \rightarrow \infty} \frac{f^{\prime}(s)}{s^{(q-4) / 2}}=0,
$$

where $2^{*}=2 N /(N-2)$.

$\left(\mathrm{f}_{3}\right)$ There exists $\theta>2$ such that

$$
0<\frac{\theta}{2} F(s) \leq s f(s), \text { for } s>0,
$$

where $F(s)=\int_{0}^{s} f(t) d t$.

$\left(\mathrm{f}_{4}\right) f^{\prime}(s)>0$, for all $s>0$.

$\left(f_{5}\right)$ There exist $q \in\left(2,2^{*}\right)$ and a constant $C>0$ such that

$$
s f(s)-F(s) \geq C|s|^{q / 2}, \quad \text { for all } s \geq 0 .
$$

We state that the magnetic field does not play any role on the number of solutions of (1) and therefore a result in the same spirit of [7] and [11] holds. More precisely, our main results are the following:

THEOREM 1.1. Suppose that $f$ satisfies $\left(\mathrm{f}_{1}\right)-\left(\mathrm{f}_{5}\right)$. There exist $\lambda^{*}>0$ such that for any $\lambda>\lambda^{*}$ problem (1.5) has at least cat $_{\Gamma_{1 \lambda}}\left(\Gamma_{1 \lambda}\right)$ nontrivial weak solutions.

To established the result in terms of Morse theory, we introduce some notation. For any $\lambda>0$, let $H_{A_{\lambda}}^{1}\left(\Omega_{\lambda}, \Gamma_{0 \lambda}\right)$ be the Hilbert space

$$
H_{A_{\lambda}}^{1}\left(\Omega_{\lambda}, \Gamma_{0 \lambda}\right) \doteq\left\{u \in L^{2}\left(\Omega_{\lambda}, \mathbb{C}\right) ;\left|\nabla_{A_{\lambda}} u\right| \in L^{2}\left(\Omega_{\lambda}\right) \text {, trace of } u=0 \text { on } \Gamma_{0 \lambda}\right\},
$$

endowed with the norm

$$
\langle u, v\rangle_{A_{\lambda}} \doteq \operatorname{Re}\left\{\int_{\Omega_{\lambda}}\left(\nabla_{A_{\lambda}} u \overline{\nabla_{A_{\lambda} v}}+u \bar{v}\right) d x\right\},
$$

where

$$
\nabla_{A_{\lambda}} u \doteq\left(D_{A_{\lambda}}^{j} u\right)_{j=1}^{N}, \quad D_{A_{\lambda}}^{j} u \doteq-i \partial_{j} u-A_{\lambda}^{j} u
$$


and $\operatorname{Re}(w)$ is the real part of $w \in \mathbb{C}$ and $\bar{w}$ is its complex conjugate. The norm induced by this inner product is given by

$$
\|u\|_{A_{\lambda}}=\left(\int_{\Omega_{\lambda}}\left(\left|\nabla_{A_{\lambda}} u\right|^{2}+|u|^{2}\right) d x\right)^{1 / 2} .
$$

By [22], we can state a version of diamagnetic inequality for the space $H_{A_{\lambda}}^{1}\left(\Omega_{\lambda}, \Gamma_{0 \lambda}\right)$ : For any $u \in H_{A_{\lambda}}^{1}\left(\Omega_{\lambda}, \Gamma_{0 \lambda}\right)$,

$$
\left|\nabla_{A_{\lambda}} u\right| \geq|\nabla| u|| \text {. }
$$

As a consequence, the embedding $H_{A_{\lambda}}^{1}\left(\Omega_{\lambda}, \Gamma_{0 \lambda}\right) \hookrightarrow L^{p}\left(\Omega_{\lambda}, \mathbb{R}\right)$ is continuous for $1 \leq p \leq 2^{*}$ and it is compact for $1 \leq p<2^{*}$. It is worth pointing out that the embedding constants do not depend on $\lambda$, because of the assumption that $\Omega \subset \mathbb{R}^{N}(N \geq 3)$ is a bounded domain with smooth boundary $\partial \Omega$. We also emphasize that the regularity on $\partial \Omega$ assumed here must be sufficient to obtain $r_{0}>0$ such that

$$
B_{r_{0}}\left(y+r_{0} \nu_{y}\right) \subset \Omega \quad \text { and } \quad B_{r_{0}}\left(y-r_{0} \nu_{y}\right) \subset \mathbb{R}^{N} \backslash \Omega,
$$

uniformly for $y \in \partial \Omega$, where $\nu_{y}$ is the inward unitary normal vector to $\partial \Omega$ in $y$ and $B_{r}(z)$ denotes the ball of radius $r$ centered at $z$.

The functional associated with (1.5) $I_{\lambda}: H_{A_{\lambda}}^{1}\left(\Omega_{\lambda}, \Gamma_{0 \lambda}\right) \rightarrow \mathbb{R}$ is given by

$$
I_{\lambda}(u) \doteq \frac{1}{2} \int_{\Omega_{\lambda}}\left(\left|\nabla_{A_{\lambda}} u\right|^{2}+|u|^{2}\right) d x-\frac{1}{2} \int_{\Omega_{\lambda}} F\left(|u|^{2}\right) d x .
$$

From conditions $\left(\mathrm{f}_{1}\right)-\left(\mathrm{f}_{2}\right)$, the functional $I_{\lambda}$ is well defined and belongs to $C^{2}\left(H_{A_{\lambda}}^{1}\left(\Omega_{\lambda}, \Gamma_{0 \lambda}\right), \mathbb{R}\right)$. Furthermore,

$$
I_{\lambda}^{\prime}(u) v \doteq \operatorname{Re}\left\{\int_{\Omega_{\lambda}} \nabla_{A_{\lambda}} u \overline{\nabla_{A_{\lambda}} v}+u \bar{v} d x-\int_{\Omega_{\lambda}} f\left(|u|^{2}\right) u \bar{v} d x\right\},
$$

for all $u, v \in H_{A_{\lambda}}^{1}\left(\Omega_{\lambda}, \Gamma_{0 \lambda}\right)$. Thus, every critical point of $I_{\lambda}$ is a weak solution of problem (1.5).

In the notation of [7], we have if $u$ is an isolated critical point of $I_{\lambda}$ and $I_{\lambda}(u)=c$, the polynomial Morse index $i_{t}(u)$ of $u$ is defined by

$$
i_{t}(u)=\sum_{k} \operatorname{dim}\left[H^{k}\left(I_{\lambda}^{c} \cap U,\left(I_{\lambda}^{c} \backslash\{u\}\right) \cap U\right)\right] t^{k},
$$

where $H^{k}(\cdot, \cdot)$ denotes the $k$ th group de homology with coefficients in some field $\mathbf{K}, U$ is a neighbourhood of $u$ and

$$
I_{\lambda}^{c}=\left\{v \in H_{A_{\lambda}}^{1}\left(\Omega_{\lambda}, \Gamma_{0 \lambda}\right) ; I_{\lambda}(v) \leq c\right\} .
$$

As is proved in [6, Theorem I.5.8], if $u$ is a non-degenerate critical point, then $i_{t}(u)=t^{\mu(t)}$, where $\mu(u)$ denotes the numeric Morse index of $u$. 
Let $X$ be a topological space. The Poincaré polynomial of $X$ is defined by

$$
\mathcal{P}_{t}(X)=\sum_{k} \operatorname{dim}\left[H^{k}(X)\right] t^{k} .
$$

Following [7], we can prove the ensuing multiplicity result:

THEOREM 1.2. Suppose that $f$ satisfies $\left(\mathrm{f}_{1}\right)-\left(\mathrm{f}_{5}\right)$ and the set $\mathcal{K}$ of nontrivial solutions of problem (1.5) is discrete. Then, there exists $\lambda^{*}>0$ such that

$$
\sum_{u \in \mathcal{K}} i_{t}(u)=t \mathcal{P}_{t}\left(\Gamma_{1 \lambda}\right)+t^{2}\left[\mathcal{P}_{t}\left(\Gamma_{1 \lambda}\right)-1\right]+(t+1) \mathcal{Q}(t),
$$

for every $\lambda>\lambda^{*}$, where $\mathcal{Q}(t)$ is a polynomial with non-negative integer coefficients.

In the non-degenerate case, we have:

Corollary 1.1. Suppose that $f$ satisfies $\left(\mathrm{f}_{1}\right)-\left(\mathrm{f}_{5}\right)$ and the solutions of problem (1.5) are non-degenerate. Then, there exists $\lambda^{*}>0$ such that

$$
\sum_{u \in \mathcal{K}} t^{\mu(u)}=t \mathcal{P}_{t}\left(\Gamma_{1 \lambda}\right)+t^{2}\left[\mathcal{P}_{t}\left(\Gamma_{1 \lambda}\right)-1\right]+(t+1) \mathcal{Q}(t),
$$

for every $\lambda>\lambda^{*}$, where $\mathcal{Q}(t)$ is a polynomial with non-negative integer coeffcients.

As observed in [7] (see also [19]), the application of the Morse theory can give better information than the use of the Lusternik-Schnirelman theorem. Theorem 1.2 shows that the problem (1.5) possesses at least $2 \mathcal{P}_{1}\left(\Gamma_{1 \lambda}\right)-1$ nontrivial weak solutions. In the case of $\Gamma_{1 \lambda}$ is topologically trivial, we have $\mathcal{P}_{1}\left(\Gamma_{1 \lambda}\right)=1$ and this theorem does not provide any additional information about multiplicity of solutions. On the other hand, when $\Gamma_{1 \lambda}$ is a topologically rich domain, for example, if $\Gamma_{1 \lambda}$ is obtained by contractible submanifold cutting off $k$ contractible open non-empty sets in $\partial \Omega$, we obtain that the number of nontrivial solutions of (1.5) is affected by $k$, even if the category of $\Gamma_{1 \lambda}$ is 2 .

In order to prove Theorems 1.1 and 1.2, we combine the Benci and Cerami approach [7] with a variation of the arguments of Candela and Lazzo [11]. The major steps in Benci and Cerami approach are the analysis of the behavior of some critical levels related to problem (3) and the comparison of the topology of $\Omega$ with some sublevel sets of the functional associated with (3). Although we use this machinery, we have to make a detailed analysis of the behavior of the minimax levels associated with the problem (1.5) and a more involved proof that the barycenter function maps suitable sublevel sets of the functional associated with (1.5) in a neighbourhood of the portion of the boundary where the Neumann condition is prescribed. This is because the equation (1.5) involves a magnetic field and mixed Dirichlet-Neumann boundary conditions. Moreover, 
as the nonlinearity is not necessarily homogeneous, our arguments are different from what can already be found in [11]. Once these crucial steps are verified, we can employ the Morse theory developed in [7, Section 5] to estimate the number of nontrivial solutions to (1.5) in terms of the topology of the part of the boundary where the Neumann condition is assumed.

Theorems 1.1 and 1.2 can be seen as a complement of the studies made in [2], [7] and [11] in the following aspects: 1) In [2] only the Dirichlet boundary condition was considered; 2) In [7], the problem was considered for the Laplacian operator and Dirichlet boundary condition. Here we are working with a more general boundary condition and with a class of operators which includes the Laplacian operator as a particular case; 3). In [11], the problem was also considered for Laplacian operator and with a homogeneous nonlinearity. In the present paper we deal with a class of nonlinearities that has the homogeneous functions as a particular case. As we are mainly considering a non homogeneous nonlinearity, our estimates are more delicate and we need to make a careful analysis in several estimates involving different arguments from those used in [11], see Sections 3,4 and 5 .

\section{The Palais-Smale condition}

In this section we establish the Palais-Smale condition for the functional $I_{\lambda}$, defined by (1.8), and for the functional $I_{\lambda}$ constrained to $M_{\lambda}$. As a direct consequence of $\left(\mathrm{f}_{1}\right)-\left(\mathrm{f}_{3}\right)$, we obtain

$\left(\mathrm{f}_{6}\right)$ Given $\varepsilon>0$, there exist constant $C_{\varepsilon}>0$ such that

$$
f(s) \leq \varepsilon+C_{\varepsilon} s^{(q-2) / 2}, \quad \text { for all } s \geq 0,
$$

where $q \in\left(2,2^{*}\right)$.

$\left(\mathrm{f}_{7}\right)$ There exists $\theta>2$ and a constant $C>0$ such that

$$
F(s) \geq C|s|^{\theta / 2}-C, \quad \text { for all } s \geq 0,
$$

where $F(s)=\int_{0}^{s} f(t) d t$.

Proposition 2.1. The functional $I_{\lambda}$ satisfies the Palais-Smale condition, that is, every sequence $\left(u_{n}\right) \subset H_{A_{\lambda}}^{1}$ for which $\sup _{n \in \mathbb{N}}\left|I_{\lambda}\left(u_{n}\right)\right|<\infty$ and $I_{\lambda}^{\prime}\left(u_{n}\right) \rightarrow 0$, as $n \rightarrow \infty$, possesses a converging subsequence.

Proof. Given a sequence $\left(u_{n}\right) \subset H_{A_{\lambda}}^{1}\left(\Omega_{\lambda}, \Gamma_{0 \lambda}\right)$ such that $\sup _{n \in \mathbb{N}}\left|I_{\lambda}\left(u_{n}\right)\right|<\infty$ and $I_{\lambda}^{\prime}\left(u_{n}\right) \rightarrow 0$, as $n \rightarrow \infty$, we may assume that $I_{\lambda}\left(u_{n}\right) \rightarrow d$ and $I_{\lambda}^{\prime}\left(u_{n}\right) \rightarrow 0$, as $n \rightarrow \infty$, for some $d \in \mathbb{R}$. We claim that $\left(u_{n}\right)$ is bounded. In fact, from $\left(\mathrm{f}_{3}\right)$, 
we have

$$
\begin{aligned}
& d+o_{n}(1)+o_{n}(1)\left\|u_{n}\right\|_{A_{\lambda}}=I_{\lambda}\left(u_{n}\right)-\frac{1}{\theta} I_{\lambda}^{\prime}\left(u_{n}\right) u_{n} \\
= & \left(\frac{1}{2}-\frac{1}{\theta}\right)\left\|u_{n}\right\|_{A_{\lambda}}^{2}+\int_{\Omega_{\lambda}}\left(\frac{1}{\theta} f\left(\left|u_{n}\right|^{2}\right)\left|u_{n}\right|^{2}-\frac{1}{2} F\left(\left|u_{n}\right|^{2}\right) \geq\left(\frac{1}{2}-\frac{1}{\theta}\right)\left\|u_{n}\right\|_{A_{\lambda}}^{2},\right.
\end{aligned}
$$

where $o_{n}(1)$ denotes a quantity going to zero zero as $n \rightarrow \infty$. From this, we obtain that $\left(u_{n}\right)$ is bounded. As a consequence, we may assume that $\left(u_{n}\right)$ has a subsequence, still denoted by $\left(u_{n}\right)$, and there exists $u \in H_{A_{\lambda}}^{1}\left(\Omega_{\lambda}, \Gamma_{0 \lambda}\right)$ such that

$$
\begin{array}{ll}
u_{n} \rightarrow u & \text { in } H_{A_{\lambda}}^{1}\left(\Omega_{\lambda}, \Gamma_{0 \lambda}\right), \\
u_{n} \rightarrow u & \text { in } L^{p}\left(\Omega_{\lambda}, \mathbb{C}\right), \text { for all } p \in\left[1,2^{*}\right), \\
u_{n} \rightarrow u & \text { a.e. in } \Omega_{\lambda} .
\end{array}
$$

Invoking the definition of $I_{\lambda}^{\prime}$, we obtain

$$
\left\|u_{n}-u\right\|_{A_{\lambda}}^{2}=\left(I_{\lambda}^{\prime}\left(u_{n}\right)-I_{\lambda}^{\prime}(u)\right)\left(u_{n}-u\right)-\operatorname{Re}\left\{\int_{\Omega_{\lambda}}\left(f\left(\left|u_{n}\right|^{2}\right) u_{n}-f\left(|u|^{2}\right) u\right) \overline{\left(u_{n}-u\right)}\right\} .
$$

Thus, from $\left(\mathrm{f}_{6}\right)$ and $(9)$,

$$
\begin{aligned}
\left\|u_{n}-u\right\|_{A_{\lambda}}^{2} \leq & \left|I_{\lambda}^{\prime}\left(u_{n}\right)\left(u_{n}-u\right)\right|+\left|I_{\lambda}^{\prime}(u)\left(u_{n}-u\right)\right| \\
& +\int_{\Omega_{\lambda}}\left|f\left(\left|u_{n}\right|^{2}\right) u_{n}-f\left(|u|^{2}\right) u\right|\left|u_{n}-u\right|=o_{n}(1),
\end{aligned}
$$

as $n \rightarrow \infty$. Hence, $u_{n} \rightarrow u$ in $H_{A_{\lambda}}^{1}\left(\Omega_{\lambda}, \Gamma_{0 \lambda}\right)$.

By $\left(f_{6}\right)-\left(f_{7}\right)$, it is a simple matter to check that $I_{\lambda}$ satisfies the geometric hypotheses of the mountain pass theorem. From this and Proposition 2.1, for any $\lambda>0$, there exists $u_{\lambda} \in H_{A_{\lambda}}^{1}\left(\Omega_{\lambda}, \Gamma_{0 \lambda}\right)$ such that $I_{\lambda}^{\prime}\left(u_{\lambda}\right)=0$ and $I_{\lambda}\left(u_{\lambda}\right) \doteq b_{\lambda}$, where $b_{\lambda}$ denotes the mountain pass level of the functional $I_{\lambda}$. From $\left(f_{4}\right)$, the level $b_{\lambda}$ satisfies (see [36])

$$
b_{\lambda}=\inf _{u \in M_{\lambda}} I_{\lambda}(u),
$$

where $M_{\lambda}$ denotes the Nehari manifold associated with $I_{\lambda}$, namely

$$
M_{\lambda}=\left\{u \in H_{A_{\lambda}}^{1}\left(\Omega_{\lambda}, \Gamma_{0 \lambda}\right) \backslash\{0\} ; I_{\Omega_{\lambda}}^{\prime}(u) u=0\right\} .
$$

Since we are intend to consider the functional $I_{\lambda}$ constrained to $M_{\lambda}$, the next two results are required.

Proposition 2.2. Suppose that $f$ satisfies $\left(\mathrm{f}_{1}\right)$ and $\left(\mathrm{f}_{2}\right)$. Then, there exists $\delta_{0}>0$ independent of $\lambda>0$ such that every $u \in M_{\lambda}$ satisfies

$$
\|u\|_{A_{\lambda}} \geq \delta_{0} \quad \text { and } \quad I_{\lambda}(u) \geq \delta_{0} .
$$


Proof. From $\left(\mathrm{f}_{6}\right)$, given $\varepsilon>0$ there exists $C_{\varepsilon}>0$ such that for every $u \in M_{\lambda}$

$$
\|u\|_{A_{\lambda}}^{2}=\int_{\Omega_{\lambda}} f\left(|u|^{2}\right)|u|^{2} \leq \varepsilon \int_{\Omega_{\lambda}}|u|_{\Omega_{\lambda}, 2}^{2}+C_{\varepsilon} \int_{\Omega_{\lambda}}|u|_{\Omega_{\lambda}, q}^{q} .
$$

Since the embedding $H_{A_{\lambda}}^{1}\left(\Omega_{\lambda}, \Gamma_{0 \lambda}\right) \hookrightarrow L^{p}\left(\Omega_{\lambda}, \mathbb{C}\right)$ is continuous for $p \in\left[1,2^{*}\right]$ and the embedding constant does not depend on $\lambda$, there exists a positive constant $C$ independent of $\lambda$ such that

$$
\|u\|_{A_{\lambda}}^{2} \leq C\left(\varepsilon\|u\|_{A_{\lambda}}^{2}+C_{\varepsilon}\|u\|_{A_{\lambda}}^{q}\right) .
$$

Taking $\varepsilon=1 /(2 C)$ in $(2.2)$, we have

$$
\|u\|_{A_{\lambda}} \geq\left(\frac{1}{2 C C_{\varepsilon}}\right)^{1 /(q-2)}=: \delta_{1}>0 .
$$

For any $u \in M_{\lambda}$, from $\left(\mathrm{f}_{3}\right)$ and $(2.3)$, it follows that

$$
\begin{aligned}
I_{\lambda}(u) & =\left(\frac{1}{2}-\frac{1}{\theta}\right)\|u\|_{A_{\lambda}}^{2}+\int_{\Omega_{\lambda}}\left(\frac{1}{\theta} f\left(|u|^{2}\right)|u|^{2}-\frac{1}{2} F\left(|u|^{2}\right)\right) \\
& \geq\left(\frac{1}{2}-\frac{1}{\theta}\right)\|u\|_{A_{\lambda}}^{2} \geq\left(\frac{1}{2}-\frac{1}{\theta}\right) \delta_{1}^{2}=: \delta_{2} .
\end{aligned}
$$

Taking $\delta_{0} \doteq \min \left\{\delta_{1}, \delta_{2}\right\}=\delta_{2}$, we conclude the proof.

Proposition 2.3. The functional $I_{\lambda}$ constrained to $M_{\lambda}$ satisfies the PalaisSmale condition.

Proof. Let $\left(u_{n}\right) \subset M_{\lambda}$ be a sequence such that $\sup _{n \in \mathbb{N}}\left|I_{\lambda}\left(u_{n}\right)\right|<\infty$ and $\left(\left.I_{\lambda}\right|_{M_{\lambda}}\right)^{\prime}\left(u_{n}\right) \rightarrow 0$, as $n \rightarrow \infty$. We can assume, by taking a subsequence if necessary, that $I_{\lambda}\left(u_{n}\right) \rightarrow d$, for some $d \in \mathbb{R}$. By [36, Proposition 5.12], for each $n \in \mathbb{N}$ there exists $\mu_{n} \in \mathbb{R}$ such that

$$
I_{\lambda}^{\prime}\left(u_{n}\right)-\mu_{n} G_{\lambda}^{\prime}\left(u_{n}\right)=\left(\left.I_{\lambda}\right|_{M_{\lambda}}\right)^{\prime}\left(u_{n}\right)=o_{n}(1),
$$

where $G_{\lambda}(v)=I_{\lambda}^{\prime}(v) v$, for all $v \in H_{A_{\lambda}}^{1}\left(\Omega_{\lambda}, \Gamma_{0 \lambda}\right)$.

As in the proof of Proposition 2.1, $\left(u_{n}\right)$ is bounded. Hence, we may suppose that $\left(u_{n}\right)$ has a subsequence, still denoted by $\left(u_{n}\right)$, and there exists $u \in$ $H_{A_{\lambda}}^{1}\left(\Omega_{\lambda}, \Gamma_{0 \lambda}\right)$ such that

$$
\begin{array}{ll}
u_{n} \rightarrow u & \text { in } H_{A_{\lambda}}^{1}\left(\Omega_{\lambda}, \Gamma_{0 \lambda}\right), \\
u_{n} \rightarrow u & \text { in } L^{p}\left(\Omega_{\lambda}, \mathbb{C}\right), \text { for all } p \in[1,2 *), \\
u_{n} \rightarrow u & \text { a.e. on } \Omega_{\lambda} .
\end{array}
$$

Since $u_{n} \in M_{\lambda}$, the condition $\left(\mathrm{f}_{4}\right)$ implies

$$
G_{\lambda}^{\prime}\left(u_{n}\right) u_{n}=-2 \int_{\Omega_{\lambda}} f^{\prime}\left(\left|u_{n}\right|^{2}\right)\left|u_{n}\right|^{4} \leq 0 .
$$


Moreover, by Proposition 2.2, we have

$$
\int_{\Omega_{\lambda}} f\left(\left|u_{n}\right|^{2}\right)\left|u_{n}\right|^{2} \geq \delta_{0}^{2}, \quad \text { for all } n \in \mathbb{N} .
$$

Taking $n \rightarrow \infty$ and using the Sobolev embedding, we obtain

$$
\int_{\Omega_{\lambda}} f\left(|u|^{2}\right)|u|^{2} \geq \delta_{0}^{2}
$$

and so, $u \not \equiv 0$. From this, (2.5) and Fatou lemma, we have

$$
\liminf _{n \rightarrow \infty} G_{\lambda}^{\prime}\left(u_{n}\right) u_{n}=\liminf _{n \rightarrow \infty}-2 \int_{\Omega_{\lambda}} f^{\prime}\left(\left|u_{n}\right|^{2}\right)\left|u_{n}\right|^{4} \leq-2 \int_{\Omega_{\lambda}} f^{\prime}\left(|u|^{2}\right)|u|^{4}<0 .
$$

Now, we use (2.4) to obtain that $\mu_{n} \rightarrow 0$, as $n \rightarrow \infty$. Consequently, the sequence $\left(u_{n}\right)$ also satisfies $\sup _{n \in \mathbb{N}}\left|I_{\lambda}\left(u_{n}\right)\right|<\infty$ and $I_{\lambda}^{\prime}\left(u_{n}\right) \rightarrow 0$, as $n \rightarrow \infty$. Proposition 2.3 now shows that the functional $I_{\lambda}$ constrained to $M_{\lambda}$ satisfies the Palais-Smale condition.

We can proceed analogously to the proof of Proposition 2.3 to show the next result.

Corollary 2.1. If $u$ is a critical of the functional $I_{\lambda}$ constrained to $M_{\lambda}$, then $u$ is a nontrivial critical point of $I_{\lambda}$.

\section{Preliminaries}

Firstly we introduce some notation. Let $\mathbb{R}_{+}^{N}=\left\{\left(x_{1}, \ldots, x_{N}\right) \in \mathbb{R}^{N}: x_{N}>0\right\}$ and $\mathbb{R}^{N-1}=\left\{\left(x_{1}, \ldots, x_{N}\right) \in \mathbb{R}^{N}: x_{N}=0\right\}$. Consider the problems

$$
-\Delta u+u=f\left(u^{2}\right) u \quad \text { in } \mathbb{R}_{+}^{N}, \quad \frac{\partial u}{\partial \nu}=0 \quad \text { on } \mathbb{R}^{N-1}
$$

and

$$
-\Delta u+u=f\left(u^{2}\right) u \quad \text { in } \mathbb{R}^{N}, \quad u \in H^{1}\left(\mathbb{R}^{N}\right) .
$$

Consider now the respective functionals associated with the above problems

$$
J_{\infty}(u) \doteq \frac{1}{2} \int_{\mathbb{R}_{+}^{N}}\left(|\nabla u|^{2}+u^{2}\right)-\frac{1}{2} \int_{\mathbb{R}_{+}^{N}} F\left(u^{2}\right), \quad \text { for all } u \in H^{1}\left(\mathbb{R}_{+}^{N}\right),
$$

and

$$
J_{\mathbb{R}^{N}}(u) \doteq \frac{1}{2} \int_{\mathbb{R}^{N}}\left(|\nabla u|^{2}+u^{2}\right)-\frac{1}{2} \int_{\mathbb{R}^{N}} F\left(u^{2}\right), \quad \text { for all } u \in H^{1}\left(\mathbb{R}^{N}\right) .
$$

We define the corresponding Nehari manifolds and mountain pass levels:

$$
N_{\infty} \doteq\left\{u \in H^{1}\left(\mathbb{R}_{+}^{N}\right) \backslash\{0\} ; J_{\infty}^{\prime}(u) u=0\right\} \quad \text { and } \quad c_{\infty} \doteq \inf _{N_{\infty}} J_{\infty}
$$


and

$$
N_{\mathbb{R}^{N}} \doteq\left\{u \in H^{1}\left(\mathbb{R}^{N}\right) \backslash\{0\} ; J_{\mathbb{R}^{N}}^{\prime}(u) u=0\right\} \quad \text { and } \quad c_{\mathbb{R}^{N}} \doteq \inf _{N_{\mathbb{R}^{N}}} J_{\mathbb{R}^{N}} .
$$

By [9], [29], (3.2) has a radially symmetric positive solution $w \in H^{1}\left(\mathbb{R}^{N}\right) \cap$ $C^{2}\left(\mathbb{R}^{N}\right)$. Moreover, the restriction of $w$ to $\mathbb{R}_{+}^{N}$ is a solution of (3.1). As a consequence,

$$
c_{\mathbb{R}^{N}}=2 c_{\infty} .
$$

Let $r>0$ be such that the sets

$$
\Gamma_{1}^{+} \doteq\left\{x \in \mathbb{R}^{N} ; \operatorname{dist}\left(x, \Gamma_{1}\right)<r\right\}, \quad \Gamma_{1}^{-} \doteq\left\{x \in \Gamma_{1} ; \operatorname{dist}\left(x, \Gamma_{0}\right) \geq r\right\}
$$

are homotopically equivalent to $\Gamma_{1}$. Let $\eta \in C^{\infty}\left(\mathbb{R}_{+}\right)$be a non-increasing function such that $\eta=1$ on $[0, r / 2], \eta=0$ on $[r,+\infty),\left|\eta^{\prime}\right| \in L^{\infty}\left(\mathbb{R}_{+}\right)$. We will denote by $\left(\Gamma_{1}^{-}\right)_{\lambda}$ the set $\lambda \Gamma_{1}^{-}$. For any $y \in\left(\Gamma_{1}^{-}\right)_{\lambda}$, we define the function

$$
x \in \Omega_{\lambda} \mapsto e^{i \tau_{\lambda, y}(x)} \eta\left(\frac{|x-y|}{\lambda}\right) w(x-y), \quad \text { where } \tau_{\lambda, y}(x) \doteq \sum_{j=1}^{N} A_{\lambda}^{j}(y) x^{j} .
$$

By definition of $\eta$, this function belongs to $H_{A_{\lambda}}^{1}\left(\Omega_{\lambda}, \Gamma_{0 \lambda}\right)$. From $\left(f_{1}\right)-\left(f_{4}\right)$, there exists $t_{\lambda, y}>0$ such that

$$
t_{\lambda, y} e^{i \tau_{\lambda, y}} \eta\left(\frac{|\cdot-y|}{\lambda}\right) w(\cdot-y) \in M_{\lambda} .
$$

Hence, $y \in\left(\Gamma_{1}^{-}\right)_{\lambda}$, and so we are able to define the function $\Phi_{\lambda}:\left(\Gamma_{1}^{-}\right)_{\lambda} \rightarrow M_{\lambda}$ by

$$
\Phi_{\lambda}(y)(x)=t_{\lambda, y} e^{i \tau_{\lambda, y}(x)} \eta\left(\frac{|x-y|}{\lambda}\right) w(x-y), \quad \text { for all } x \in \Omega_{\lambda} .
$$

Proposition 3.1. Suppose that $f$ satisfies $\left(f_{1}\right)$ and $\left(f_{2}\right)$. Then, the limit holds:

$$
\lim _{\lambda \rightarrow+\infty} \max _{y \in \Gamma_{1 \lambda}^{-}}\left|I_{\lambda}\left(\Phi_{\lambda}(y)\right)-c_{\infty}\right|=0 .
$$

Proof. Let $\left(\lambda_{n}\right)$ be any sequence such that $\lambda_{n} \rightarrow \infty$, as $n \rightarrow \infty$. Since $\left(\Gamma_{1}^{-}\right)_{\lambda_{n}}$ is a compact set and $I_{\lambda_{n}}\left(\Phi_{\lambda_{n}}\right) \in C\left(\left(\Gamma_{1}^{-}\right)_{\lambda_{n}}\right)$, it suffices to prove that

$$
\lim _{n \rightarrow \infty} I_{\lambda_{n}}\left(\Phi_{\lambda_{n}}\left(y_{n}\right)\right)=c_{\infty}
$$

for $y_{n} \in\left(\Gamma_{1}^{-}\right)_{\lambda_{n}}$ where the function $\left|I_{\lambda_{n}}\left(\Phi_{\lambda_{n}}(\cdot)\right)-c_{\infty}\right|$ attains its maximum on $\left(\Gamma_{1}^{-}\right)_{\lambda_{n}}$. By definition of $\nabla_{A_{\lambda_{n}}}$, for any $y \in\left(\Gamma_{1}^{-}\right)_{\lambda_{n}}$, we have

$$
\left|\nabla_{A_{\lambda_{n}}} \Phi_{\lambda_{n}}(y)\right|^{2}=\sum_{j=1}^{N}\left|D_{A_{\lambda_{n}}}^{j}\left(\Phi_{\lambda_{n}}(y)\right)\right|^{2},
$$


where $D_{A_{\lambda_{n}}}^{j}\left(\Phi_{\lambda_{n}}(y)(x)\right)$ are defined for $x \in \Omega_{\lambda}$ by

$$
\begin{aligned}
D_{A_{\lambda_{n}}}^{j}\left(\Phi_{\lambda_{n}}(y)(x)\right)= & -i \partial_{j} \Phi_{\lambda_{n}}(y)(x)-A^{j}\left(\frac{x}{\lambda_{n}}\right) \Phi_{\lambda_{n}}(y)(x) \\
= & t_{\lambda_{n}, y} e^{i \tau_{\lambda_{n}, y(x)}}\left[\eta\left(\frac{|x-y|}{\lambda_{n}}\right) w(x-y)\left(A^{j}\left(\frac{y}{\lambda_{n}}\right)-A^{j}\left(\frac{x}{\lambda_{n}}\right)\right)\right. \\
& \left.-i \partial_{j}\left(\eta\left(\frac{|x-y|}{\lambda_{n}}\right) w(x-y)\right)\right] .
\end{aligned}
$$

Hence,

$$
\begin{aligned}
\left|\nabla_{A_{\lambda}} \Phi_{\lambda_{n}}(y)(x)\right|^{2}= & t_{\lambda_{n}, y}^{2}\left[\eta^{2}\left(\frac{|x-y|}{\lambda_{n}}\right) w^{2}(x-y)\left|A\left(\frac{y}{\lambda_{n}}\right)-A\left(\frac{x}{\lambda_{n}}\right)\right|^{2}\right. \\
& \left.+\left|\nabla\left(\eta\left(\frac{|x-y|}{\lambda_{n}}\right) w(x-y)\right)\right|^{2}\right] .
\end{aligned}
$$

Thereby, for any $y \in\left(\Gamma_{1}^{-}\right)_{\lambda_{n}}$,

$$
\begin{aligned}
I_{\lambda_{n}}\left(\Phi_{\lambda_{n}}(y)\right)= & \frac{t_{\lambda_{n}, y}^{2}}{2} \int_{\Omega_{\lambda_{n}}}\left\{\eta^{2}\left(\frac{|x-y|}{\lambda_{n}}\right) w^{2}(x-y)\left|A\left(\frac{y}{\lambda_{n}}\right)-A\left(\frac{x}{\lambda_{n}}\right)\right|^{2}\right. \\
& \left.+\left|\nabla\left(\eta\left(\frac{|x-y|}{\lambda_{n}}\right) w(x-y)\right)\right|^{2}+\eta^{2}\left(\frac{|x-y|}{\lambda_{n}}\right) w^{2}(x-y)\right\} d x \\
& -\int_{\Omega_{\lambda_{n}}} F\left(t_{\lambda_{n}, y}^{2}\left|\eta\left(\frac{|x-y|}{\lambda_{n}}\right) w(x-y)\right|^{2}\right) d x .
\end{aligned}
$$

Let $T_{y}$ be an orthogonal operator on $\mathbb{R}^{N}$ which represents a rotation such that the unitary normal vector to $T_{y}\left(\Omega_{\lambda_{n}}-y\right)$ is $e_{N}=(0, \ldots, 1)$. Set $\widetilde{\Omega}_{\lambda_{n}, y} \doteq T_{y}\left(\Omega_{\lambda_{n}}-y\right)$. After the change of variable $z=x-y$ and using that $\eta\left(|\cdot| / \lambda_{n}\right)$ and $w$ are radially symmetric and $T_{y}$ is a rotation, we find

$$
\begin{aligned}
I_{\lambda_{n}}\left(\Phi_{\lambda_{n}}(y)\right)= & \frac{t_{\lambda_{n}, y}^{2}}{2} \int_{\widetilde{\Omega}_{\lambda_{n}, y}}\left[\eta^{2}\left(\frac{|z|}{\lambda_{n}}\right)\left[w^{2}(z)+|(\nabla w)(z)|^{2}\right]\right] d z \\
& -\frac{1}{2} \int_{\widetilde{\Omega}_{\lambda_{n}, y}} F\left(t_{\lambda_{n}, y}^{2} \eta^{2}\left(\frac{|z|}{\lambda_{n}}\right) w^{2}(z)\right) d z \\
& +\frac{t_{\lambda_{n}, y}^{2}}{2} \int_{\widetilde{\Omega}_{\lambda_{n}, y}}\left|A\left(\frac{y}{\lambda_{n}}\right)-A\left(\frac{T_{y}^{-1} z+y}{\lambda_{n}}\right)\right|^{2} \eta^{2}\left(\frac{|z|}{\lambda_{n}}\right) w^{2}(z) d z \\
& +\frac{t_{\lambda_{n}, y}^{2}}{2} \int_{\widetilde{\Omega}_{\lambda_{n}, y}} \frac{1}{\lambda_{n}{ }^{2}}\left|\eta^{\prime}\left(\frac{|z|}{\lambda_{n}}\right)\right|^{2} w^{2}(z) d z \\
& +\frac{t_{\lambda_{n}, y}^{2}}{2} \int_{\widetilde{\Omega}_{\lambda_{n}, y}} \frac{2}{\lambda_{n}}\left|\eta^{\prime}\left(\frac{|z|}{\lambda_{n}}\right)\right| w(z) \eta\left(\frac{|z|}{\lambda_{n}}\right)|\nabla w(z)| d z .
\end{aligned}
$$

We claim that the respective integrals in (3.5), (3.6) and (3.7) go to zero as $n \rightarrow+\infty$. Indeed, we first examine (3.5). Since $w \in L^{2}\left(\mathbb{R}^{N}\right)$, there exists $M>0$ 
such that

$$
\int_{\widetilde{\Omega}_{\lambda_{n}, y} \cap B_{M}^{c}(0)}\left|A\left(\frac{y}{\lambda_{n}}\right)-A\left(\frac{T_{y}^{-1} z+y}{\lambda_{n}}\right)\right|^{2} \eta^{2}\left(\frac{|z|}{\lambda_{n}}\right) w^{2}(z) d z<\varepsilon .
$$

On the other hand, since $A$ in uniformly continuous on the compact set $\bar{\Omega}$, there exists $\gamma>0$ such that

$$
|A(x+v)-A(x)|<\varepsilon, \quad \text { for all }|v| \leq \gamma, \text { for all } x \in \bar{\Omega} .
$$

Since $\left|T_{y}^{-1} z\right| \leq M$ for all $z \in \widetilde{\Omega}_{\lambda_{n}, y} \cap B_{M}(0)$, there exists $\lambda_{n}>0$ sufficiently large such that $\left|T_{y}^{-1} z / \lambda_{n}\right| \leq \gamma$, hence that, by (3.9), we have

$$
\left|A\left(\frac{y+T_{y}^{-1} z}{\lambda_{n}}\right)-A\left(\frac{y}{\lambda_{n}}\right)\right|<\varepsilon, \quad \text { for all } y \in\left(\Gamma_{1}^{-}\right)_{\lambda_{n}},
$$

for every $\lambda_{n}>0$ sufficiently large. Thus, for every $z \in B_{M}(0)$,

$$
\eta^{2}\left(\frac{|z|}{\lambda_{n}}\right) w^{2}\left|A\left(\frac{y+T_{y}^{-1} z}{\lambda_{n}}\right)-A\left(\frac{y}{\lambda_{n}}\right)\right|^{2} \chi_{\widetilde{\Omega}_{\lambda_{n}, y} \cap B_{M}(0)}(z) \leq \varepsilon^{2}|w|_{\infty, \mathbb{R}^{N}}^{2},
$$

and so

(3.10) $\lim _{\lambda_{n} \rightarrow \infty} \int_{\widetilde{\Omega}_{\lambda_{n}, y} \cap B_{M}(0)}\left|A\left(\frac{y}{\lambda_{n}}\right)-A\left(\frac{T_{y}^{-1} z+y}{\lambda_{n}}\right)\right|^{2} \eta^{2}\left(\frac{|z|}{\lambda_{n}}\right) w^{2}(z) d z=0$.

Combining (3.8) with (3.10), gives that the integral in (3.5) goes to zero as $\lambda_{n} \rightarrow \infty$. In order to analyze the integrals in (3.6)-(3.7), take a constant $C>0$ such that

$$
\begin{aligned}
\chi_{\widetilde{\Omega}_{\lambda_{n}, y}} \frac{1}{\lambda_{n}}\left(\frac{1}{2 \lambda_{n}}\left|\eta^{\prime}\left(\frac{|\cdot|}{\lambda_{n}}\right)\right|^{2} w^{2}+\left|\eta^{\prime}\left(\frac{|\cdot|}{\lambda_{n}}\right)\right| \eta\left(\frac{|\cdot|}{\lambda_{n}}\right) w|\nabla w|\right) \\
\leq C\left[w^{2}+w|\nabla w|\right] \in L^{1}\left(\mathbb{R}^{N}\right)
\end{aligned}
$$

and

$$
\begin{aligned}
\chi_{\widetilde{\Omega}_{\lambda_{n}, y}}(z) \frac{1}{\lambda_{n}}\left(\frac{1}{2 \lambda_{n}}\left|\eta^{\prime}\left(\frac{|z|}{\lambda_{n}}\right)\right|^{2} w^{2}(z)+\right. & \left.\left|\eta^{\prime}\left(\frac{|z|}{\lambda_{n}}\right)\right| \eta\left(\frac{|z|}{\lambda_{n}}\right) w(z)|\nabla w(z)|\right) \\
& \leq \frac{C}{\lambda_{n}}\left[|w(z)|^{2}+|w(z)||\nabla w(z)|\right] \rightarrow 0,
\end{aligned}
$$

almost everywhere $z \in \mathbb{R}^{N}$, as $n \rightarrow \infty$. By Lebesgue's dominated converge theorem, it follows that the integrals in (3.6) and (3.7) go to zero as $\lambda_{n} \rightarrow+\infty$. Consequently,

$$
\begin{aligned}
I_{\lambda_{n}}\left(\Phi_{\lambda_{n}}(y)\right)= & \frac{t_{\lambda_{n}, y}^{2}}{2} \int_{\widetilde{\Omega}_{\lambda_{n}, y}} \eta^{2}\left(\frac{|z|}{\lambda_{n}}\right)\left[|w|^{2}+|\nabla w|^{2}\right] d z \\
& -\frac{1}{2} \int_{\widetilde{\Omega}_{\lambda_{n}, y}} F\left(t_{\lambda_{n}, y}^{2} \eta^{2}\left(\frac{|z|}{\lambda_{n}}\right)|w|^{2}\right) d z+t_{\lambda_{n}, y}^{2} o_{\lambda_{n}}(1),
\end{aligned}
$$


where $o_{\lambda_{n}}$ (1) denotes a quantity going to zero as $n \rightarrow \infty$. Taking $y=y_{n}$ and using the notation, $\Omega_{n}=\Omega_{\lambda_{n}}, \widetilde{\Omega}_{n}=\widetilde{\Omega}_{\lambda_{n}, y_{n}}, t_{n}=t_{\lambda_{n}, y_{n}}$, we get

$$
\begin{aligned}
I_{\lambda_{n}}\left(\Phi_{\lambda_{n}}\left(y_{n}\right)\right)= & \frac{t_{n}^{2}}{2} \int_{\widetilde{\Omega}_{n}} \eta^{2}\left(\frac{|z|}{\lambda_{n}}\right)\left[|(\nabla w)(z)|^{2}+w^{2}(z)\right] d z \\
& -\frac{1}{2} \int_{\widetilde{\Omega}_{n}} F\left(t_{n}^{2} \eta^{2}\left(\frac{|z|}{\lambda_{n}}\right) w^{2}(z)\right) d z+o_{n}(1) t_{n}^{2} .
\end{aligned}
$$

We claim that $t_{n} \rightarrow 1$, as $n \rightarrow \infty$. In fact, combining the definition of $t_{n}$ with the argument used in the study of the integrals (3.5)-(3.7), yields

$$
\begin{aligned}
o_{n}(1)+\int_{\widetilde{\Omega}_{n}} \eta^{2}\left(\frac{|z|}{\lambda_{n}}\right)\left[|\nabla w|^{2}\right. & \left.+w^{2}\right] d z \\
& =\int_{\widetilde{\Omega}_{n}} f\left(t_{n}^{2} \eta^{2}\left(\frac{|z|}{\lambda_{n}}\right) w^{2}\right) \eta^{2}\left(\frac{|z|}{\lambda_{n}}\right) w^{2} d z .
\end{aligned}
$$

To establish the boundedness of $\left(t_{n}\right)$, suppose by contradiction that there exists a subsequence $t_{n_{i}} \rightarrow+\infty$. Using $\left(f_{3}\right)-\left(f_{5}\right),(1.7)$, Fatou lemma, $w>0$ in $\mathbb{R}^{N}$ and (3.12), we have

$$
\begin{aligned}
+\infty & >\int_{\mathbb{R}^{N}}\left(|\nabla w|^{2}+w^{2}\right)=\lim _{i \rightarrow \infty} \int_{\widetilde{\Omega}_{n_{i}}} \eta^{2}\left(\frac{|z|}{\lambda_{n_{i}}}\right)\left(|\nabla w(z)|^{2}+w^{2}(z)\right) d z \\
& \geq \lim _{i \rightarrow \infty} \int_{B_{r_{0}}\left(r_{0} e_{N}\right)} f\left(t_{n}^{2} \eta^{2}\left(\frac{|z|}{\lambda_{n_{i}}}\right) w^{2}(z)\right) \eta^{2}\left(\frac{|z|}{\lambda_{n_{i}}}\right) w^{2}(z) d z \\
& =\lim _{i \rightarrow \infty} \int_{B_{r_{0}}\left(r_{0} e_{N}\right)} f\left(t_{n_{i}}^{2} w^{2}(z)\right) w^{2}(z) d z=+\infty,
\end{aligned}
$$

which is impossible. Hence, $\left(t_{n}\right)$ is a bounded sequence. We can clearly assume that $t_{n} \rightarrow t_{0}$, as $n \rightarrow \infty$. To verify that $t_{0}>0$, suppose by contradiction that $t_{0}=0$. By $\left(\mathrm{f}_{1}\right)-\left(\mathrm{f}_{2}\right)$ and Lebesgue's dominated convergence, we obtain

$$
\lim _{n \rightarrow \infty} \int_{\widetilde{\Omega}_{n}} f\left(t_{n}^{2} \eta^{2}\left(\frac{|z|}{\lambda_{n}}\right) w^{2}(z)\right) \eta^{2}\left(\frac{|z|}{\lambda_{n}}\right) w^{2}(z) d z=0
$$

On the other hand, from (3.13), (3.12) and (1.7), we have

$$
\begin{aligned}
0 & <\int_{\mathbb{R}^{N}}\left(|\nabla w|^{2}+w^{2}\right) d z=\lim _{n \rightarrow \infty} \int_{\widetilde{\Omega}_{n}} \eta^{2}\left(\frac{|z|}{\lambda_{n}}\right)\left(|\nabla w(z)|^{2}+w^{2}(z)\right) d z \\
& =\lim _{n \rightarrow \infty} \int_{\widetilde{\Omega}_{n}} f\left(t_{n}^{2} \eta^{2}\left(\frac{|z|}{\lambda_{n}}\right) w^{2}(z)\right) \eta^{2}\left(\frac{|z|}{\lambda_{n}}\right) w^{2}(z) d z=0,
\end{aligned}
$$


which is a contradiction. Hence, $t_{n} \rightarrow t_{0}>0$, as $n \rightarrow \infty$. Now observe that

$$
\begin{aligned}
\int_{\mathbb{R}^{N}}\left(|\nabla w|^{2}+w^{2}\right) d z & =\lim _{n \rightarrow \infty} \int_{\widetilde{\Omega}_{n}} \eta^{2}\left(t_{n}^{2} \frac{|z|}{\lambda_{n}}\right)\left[|\nabla w(z)|^{2}+w^{2}(z)\right] d z \\
& =\lim _{n \rightarrow \infty} \int_{\widetilde{\Omega}_{n}} f\left(t_{n}^{2} \eta^{2}\left(\frac{|z|}{\lambda_{n}}\right) w^{2}(z)\right) \eta^{2}\left(\frac{|z|}{\lambda_{n}}\right) w^{2}(z) d z \\
& =\int_{\mathbb{R}^{N}} f\left(t_{0}^{2} w^{2}\right) w^{2} d z .
\end{aligned}
$$

Using this, $\left(\mathrm{f}_{4}\right)$ and the properties on $w$, we conclude that $t_{0}=1$. Therefore, the proposition follows from (3.11) and the Lebesgue's dominated convergence.

Finally, we establish a version of Lions lemma, whose proof proceeds along the same lines as in [35, Lemma 2.1] combined with interpolation of the $L^{p}$ spaces.

Lemma 2.1. Let $l>0,2 \leq s<2^{*}$ and $\lambda_{n} \rightarrow+\infty$. Let $\left\{u_{n}\right\} \subset H^{1}\left(\Omega_{\lambda_{n}}\right)$ be a sequence such that

$$
\lim _{n \rightarrow \infty} \sup _{y \in \mathbb{R}^{N}} \int_{B_{l}(y) \cap \Omega_{\lambda_{n}}}\left|u_{n}\right|^{s} d x=0 .
$$

Then, for every $m \in\left(2,2^{*}\right)$,

$$
\lim _{n \rightarrow \infty} \int_{\Omega_{\lambda_{n}}}\left|u_{n}\right|^{m} d x=0 .
$$

\section{The behavior of the minimax levels}

Taking $b_{\lambda}$ given by $(2.1)$, we have:

Proposition 4.1. $\lim _{\lambda \rightarrow \infty} b_{\lambda}=c_{\infty}$.

The proof of Proposition 4.1 is long and will be carried out in a series of steps. First, by definition of $\Phi_{\lambda}(y)$ and Proposition 3.1,

$$
b_{\lambda} \leq I_{\lambda}\left(\Phi_{\lambda}(y)\right)=o_{\lambda}(1)+c_{\infty} .
$$

We now consider the auxiliary problems:

$$
\begin{cases}-\Delta u+u=f\left(u^{2}\right) u & \text { in } \Omega_{\lambda}, \\ \frac{\partial u}{\partial \nu}=0 & \text { on } \Gamma_{1 \lambda}, \\ u=0 & \text { on } \Gamma_{0 \lambda}\end{cases}
$$

and

$$
\begin{cases}-\Delta u+u=f\left(u^{2}\right) u & \text { in } \Omega_{\lambda}, \\ \frac{\partial u}{\partial \nu}=0 & \text { on } \partial \Omega_{\lambda} .\end{cases}
$$


We will denote by $H^{1}\left(\Omega_{\lambda}, \Gamma_{0 \lambda}\right)$ be the Hilbert space

$$
H^{1}\left(\Omega_{\lambda}, \Gamma_{0 \lambda}\right) \doteq\left\{u \in H^{1}\left(\Omega_{\lambda}\right) ; \text { trace of } u=0 \text { on } \Gamma_{0 \lambda}\right\},
$$

endowed with the norm

$$
\|u\|_{\Omega_{\lambda}}=\left(\int_{\Omega_{\lambda}}\left(\left|\nabla_{A_{\lambda}} u\right|^{2}+|u|^{2}\right) d x\right)^{1 / 2} .
$$

Let $J_{\lambda}: H^{1}\left(\Omega_{\lambda}, \Gamma_{0 \lambda}\right) \rightarrow \mathbb{R}$ be the functional associated with (4.2) and given by

$$
J_{\lambda}(u)=\frac{1}{2} \int_{\Omega_{\lambda}}\left(|\nabla u|^{2}+u^{2}\right) d x-\frac{1}{2} \int_{\Omega_{\lambda}} F\left(u^{2}\right) d x, \quad \text { for all } u \in H^{1}\left(\Omega_{\lambda}, \Gamma_{0 \lambda}\right) .
$$

We define the functional $\bar{J}_{\lambda}: H^{1}\left(\Omega_{\lambda}\right) \rightarrow \mathbb{R}$ associated with (4.3) by

$$
\bar{J}_{\lambda}(u)=\frac{1}{2} \int_{\Omega_{\lambda}}\left(|\nabla u|^{2}+u^{2}\right) d x-\frac{1}{2} \int_{\Omega_{\lambda}} F\left(u^{2}\right) d x, \quad \text { for all } u \in H^{1}\left(\Omega_{\lambda}\right),
$$

with corresponding Nehari manifold and mountain pass level given by

$$
\bar{N}_{\lambda} \doteq\left\{u \in H^{1}\left(\Omega_{\lambda}\right) \backslash\{0\} ; \bar{J}_{\lambda}^{\prime}(u) u=0\right\} \quad \text { and } \quad \bar{c}_{\lambda} \doteq \inf _{\bar{N}_{\lambda}} \bar{J}_{\lambda} .
$$

We will also denote by $c_{\lambda}$ the mountain pass level associated with the problem (4.2). By the definition of these levels and from (1.6), we find

$$
b_{\lambda} \geq c_{\lambda} \geq \bar{c}_{\lambda}>0 .
$$

From (4.1)-(4.4), we deduce that it suffices to show that

$$
\lim _{\lambda \rightarrow \infty} \bar{c}_{\lambda}=c_{\infty}
$$

In order to prove (4.5), we begin by observing that the mountain pass theorem combined with a similar argument employed in the proof of Proposition 2.1 implies that there is a solution $u_{\lambda} \in H^{1}\left(\Omega_{\lambda}\right)$ of (4.3) satisfying

$$
\bar{J}_{\lambda}\left(u_{\lambda}\right)=\bar{c}_{\lambda}=\inf _{\bar{N}_{\lambda}} \bar{J}_{\lambda}, \quad \bar{J}_{\lambda}^{\prime}\left(u_{\lambda}\right)=0,
$$

for every $\lambda>0$. Combining (4.4) with (4.6), gives that $\sup _{\lambda>0} \bar{J}_{\lambda}\left(u_{\lambda}\right)<\infty$ and $\bar{J}_{\lambda}^{\prime}\left(u_{\lambda}\right) u_{\lambda}=0$ for all $\lambda>0$. By $\left(f_{3}\right)$,

$$
\sup _{\lambda>0}\left\|u_{\lambda}\right\|_{\Omega_{\lambda}}<\infty
$$

(where $\|\cdot\|_{\Omega_{\lambda}}$ denotes the norm of $H^{1}\left(\Omega_{\lambda}\right)$ ). Exploiting similar argument used in the proof of Proposition 2.2, we may assume that

$$
\left\|u_{\lambda}\right\|_{\Omega_{\lambda}}^{2} \geq \delta_{0} \quad \text { and } \quad \bar{J}_{\lambda}\left(u_{\lambda}\right)=\bar{c}_{\lambda} \geq \delta_{0}, \quad \text { for all } \lambda>0,
$$


for some constant $\delta_{0}>0$ independent of $\lambda$. From (4.8) and Lemma 3.1, there exist $\left(y_{\lambda}\right)_{\lambda} \subset \mathbb{R}^{N}, l>0$ and $\gamma>0$ such that

$$
\liminf _{\lambda \rightarrow \infty} \int_{\Omega_{\lambda} \cap B_{l}\left(y_{\lambda}\right)}\left|u_{\lambda}\right|^{2} d x \geq \gamma>0 .
$$

Moreover, by increasing $l$ if necessary, we may assume that $y_{\lambda} \in \Omega_{\lambda}$ for every $\lambda>0$, because (4.9) yileds $\Omega_{\lambda} \cap B_{l}\left(y_{\lambda}\right) \neq \emptyset$, for every $\lambda$.

Lemma 4.1. There exists a constant $C>0$ such that $\operatorname{dist}\left(y_{\lambda}, \partial \Omega_{\lambda}\right) \leq C$, for every $\lambda>0$.

Proof. Suppose the lemma were false. Then, we could find a sequence $\left(\lambda_{n}\right)$ such that $\lambda_{n} \rightarrow \infty$ and $\operatorname{dist}\left(y_{\lambda_{n}}, \partial \Omega_{\lambda_{n}}\right) \rightarrow \infty$, as $n \rightarrow \infty$. Let $R>l$ be an arbitrary number. For $n$ sufficiently large, we have $B_{2 R}\left(y_{\lambda_{n}}\right) \subset \Omega_{\lambda_{n}}$. Define

$$
w_{\lambda_{n}, R}(x) \doteq \eta\left(\frac{|x|}{R}\right) u_{\lambda_{n}}\left(x+y_{\lambda_{n}}\right), \quad \text { for all } x \in \Omega_{\lambda_{n}}-\left\{y_{\lambda_{n}}\right\},
$$

where $\eta \in C^{\infty}(\mathbb{R})$ is such that $\eta=1$, on $[0,1], \eta=0$, on $(2,+\infty), 0 \leq \eta \leq 1$ and $\eta^{\prime} \in L^{\infty}(\mathbb{R})$. Hence, $\operatorname{supp} w_{\lambda_{n}, R} \subset B_{2 R}(0)$. We can assume that $w_{\lambda_{n}, R} \in$ $H^{1}\left(\mathbb{R}^{N}\right)$ and also $\sup _{n}\left\|w_{\lambda_{n}, R}\right\| \leq C$, for some constant $C>0$ independent $R$. Observing that

$$
\int_{B_{l}(0)}\left|w_{\lambda_{n}, R}\right|^{2} d x=\int_{B_{l}(0)}\left|u_{\lambda_{n}}\left(x+y_{\lambda_{n}}\right)\right|^{2} d x=\int_{B_{l}\left(y_{\lambda_{n}}\right)}\left|u_{\lambda_{n}}\right|^{2} d x \geq \gamma>0,
$$

we get a nontrivial function $w_{R} \in H^{1}\left(\mathbb{R}^{N}\right)$ such that

$$
\begin{aligned}
& w_{\lambda_{n}, R} \rightarrow w_{R}, \quad \text { weakly in } H^{1}\left(\mathbb{R}^{N}\right), \text { as } n \rightarrow \infty, \\
& w_{\lambda_{n}, R} \rightarrow w_{R}, \quad \text { strongly in } L_{\mathrm{loc}}^{p}\left(\mathbb{R}^{N}\right), p \in\left[1,2^{*}\right), \text { as } n \rightarrow \infty, \\
& \int_{B_{l}(0)}\left|w_{R}\right|^{2} \geq \gamma>0 .
\end{aligned}
$$

Let $\|\cdot\|$ denote the norm in of $H^{1}\left(\mathbb{R}^{N}\right)$. Since $\left\|w_{R}\right\| \leq \liminf _{n \rightarrow \infty}\left\|w_{\lambda_{n}, R}\right\|$, the family $\left(w_{R}\right)_{R} \subset H^{1}\left(\mathbb{R}^{N}\right)$ is bounded. Hence, there exists $v \in H^{1}\left(\mathbb{R}^{N}\right)$ such that

$$
\begin{aligned}
& w_{R} \rightarrow v, \quad \text { weakly in } H^{1}\left(\mathbb{R}^{N}\right), \text { as } R \rightarrow \infty, \\
& w_{R} \rightarrow v, \quad \text { strongly in } L_{\mathrm{loc}}^{p}\left(\mathbb{R}^{N}\right), p \in\left[1,2^{*}\right), \text { as } R \rightarrow \infty, \\
& \int_{B_{l}(0)}|v|^{2} \geq \gamma>0 .
\end{aligned}
$$

In particular, $v \not \equiv 0$. We assert that $v$ is a solution of (3.2). In fact, given $\phi \in C_{c}^{\infty}\left(\mathbb{R}^{N}\right)$, we take $t>0$ such that $\operatorname{supp} \phi \subset B_{t}(0)$ and $B_{t}\left(y_{\lambda_{n}}\right) \subset \Omega_{\lambda_{n}}$ for $n$ 
sufficiently large. As $u_{\lambda_{n}}$ is a weak solution of (4.3) for $\lambda=\lambda_{n}$, we have

$$
\begin{aligned}
\int_{B_{t}(0)}\left[\nabla u_{\lambda_{n}}(x\right. & \left.\left.+y_{\lambda_{n}}\right) \nabla \phi+u_{\lambda_{n}}\left(x+y_{\lambda_{n}}\right) \phi\right] \\
& =\int_{\Omega_{\lambda_{n}}}\left[\nabla u_{\lambda_{n}}\left(x+y_{\lambda_{n}}\right) \nabla \phi+u_{\lambda_{n}}\left(x+y_{\lambda_{n}}\right) \phi\right] \\
& =\int_{\Omega_{\lambda_{n}}} f\left(u_{\lambda_{n}}^{2}\left(x+y_{\lambda_{n}}\right)\right) u_{\lambda_{n}}\left(x+y_{\lambda_{n}}\right) \phi \\
& =\int_{B_{t}(0)} f\left(u_{\lambda_{n}}^{2}\left(x+y_{\lambda_{n}}\right)\right) u_{\lambda_{n}}\left(x+y_{\lambda_{n}}\right) \phi .
\end{aligned}
$$

For $n$ sufficiently large and $R>t$, we obtain

$$
\int_{B_{t}(0)}\left[\nabla w_{\lambda_{n}, R} \nabla \phi+w_{\lambda_{n}, R} \phi\right] d x=\int_{B_{t}(0)} f\left(w_{\lambda_{n}, R}^{2}\right) w_{\lambda_{n}, R} \phi d x .
$$

Taking $n \rightarrow \infty$, we have

$$
\int_{B_{t}(0)}\left[\nabla w_{R} \nabla \phi+w_{R} \phi\right] d x=\int_{B_{t}(0)} f\left(w_{R}^{2}\right) w_{R} \phi d x .
$$

Using that $\operatorname{supp} \phi \subset B_{t}(0)$ and $R>t$, we find after taking $R \rightarrow \infty$

$$
\int_{\mathbb{R}^{N}}[\nabla v \nabla \phi+v \phi]=\int_{B_{t}(0)}[\nabla v \nabla \phi+v \phi]=\int_{B_{t}(0)} f\left(v^{2}\right) v \phi=\int_{\mathbb{R}^{N}} f\left(v^{2}\right) v \phi .
$$

Since $\phi \in C_{c}^{\infty}\left(\mathbb{R}^{N}\right)$ is arbitrary, we conclude that $v$ is a nontrivial solution of (3.2). Given $M>R$, we take $n$ sufficiently large such that $B_{M}\left(y_{\lambda_{n}}\right) \subset \Omega_{\lambda_{n}}$. By (4.1)-(4.4),

$$
\begin{aligned}
o_{\lambda_{n}}(1)+c_{\infty} & \geq \bar{c}_{\lambda_{n}}=\bar{J}_{\lambda_{n}}\left(u_{\lambda_{n}}\right)-\frac{1}{2} \bar{J}_{\lambda_{n}}^{\prime}\left(u_{\lambda_{n}}\right) u_{\lambda_{n}} \\
& =\frac{1}{2} \int_{\Omega_{\lambda_{n}}}\left[f\left(u_{\lambda_{n}}^{2}\right) u_{\lambda_{n}}^{2}-F\left(u_{\lambda_{n}}^{2}\right)\right] d x \\
& \geq \frac{1}{2} \int_{B_{M}\left(y_{\lambda_{n}}\right)}\left[f\left(u_{\lambda_{n}}^{2}\right) u_{\lambda_{n}}^{2}-F\left(u_{\lambda_{n}}^{2}\right)\right] d x \\
& =\frac{1}{2} \int_{B_{M}(0)}\left[f\left(w_{\lambda_{n}, R}^{2}\right) w_{\lambda_{n}, R}^{2}-F\left(w_{\lambda_{n}, R}^{2}\right)\right] d x .
\end{aligned}
$$

By Fatou's lemma and (3.3), we obtain, after taking $n \rightarrow \infty, R \rightarrow \infty$ and $M \rightarrow \infty$,

$$
c_{\infty} \geq \frac{1}{2} \int_{\mathbb{R}^{N}}\left[f\left(v^{2}\right) v^{2}-F\left(v^{2}\right)\right] d x=J_{\mathbb{R}^{N}}(v) \geq c_{\mathbb{R}^{N}}=2 c_{\infty},
$$

which is a contradiction. Lemma 4.1 is proved.

From Lemma 4.1, by increasing $l$ if necessary, we may assume that $y_{\lambda} \in \partial \Omega_{\lambda}$ in (4.9). Let $T_{y_{\lambda}}$ be an orthogonal operator on $\mathbb{R}^{N}$ which represents a rotation 
such that the inward unitary normal vector to $\widetilde{\Omega}_{\lambda} \doteq T_{y_{\lambda}}\left(\Omega_{\lambda}-y_{\lambda}\right)$ is $e_{N}=$ $(0, \ldots, 1)$. We define

$$
v_{\lambda}(x)=u_{\lambda}\left(T_{y_{\lambda}}^{-1} x+y_{\lambda}\right), \quad \text { for all } x \in \widetilde{\Omega}_{\lambda} .
$$

In the following, we gather the properties satisfied by $v_{\lambda}$ :

(a) Since $\left\|v_{\lambda}\right\|_{\tilde{\Omega}_{\lambda}}=\left\|u_{\lambda}\right\|_{\Omega_{\lambda}}$, (4.7) shows that $\sup _{\lambda>0}\left\|v_{\lambda}\right\|_{\tilde{\Omega}_{\lambda}}<\infty$;

(b) $\int_{\widetilde{\Omega}_{\lambda}} F\left(v_{\lambda}^{2}\right) d x=\int_{\Omega_{\lambda}} F\left(u_{\lambda}^{2}\right) d x$;

(c) Since $u_{\lambda}$ is a solution of (4.3), $v_{\lambda}$ is a solution of

$$
-\Delta u+u=f\left(u^{2}\right) u \quad \text { in } \widetilde{\Omega}_{\lambda}, \quad \frac{\partial u}{\partial \nu}=0 \quad \text { on } \partial \widetilde{\Omega}_{\lambda} ;
$$

(d) $J_{\widetilde{\Omega}_{\lambda}}\left(v_{\lambda}\right)=\bar{c}_{\widetilde{\Omega}_{\lambda}}=\bar{c}_{\lambda}$, where $J_{\widetilde{\Omega}_{\lambda}}$ is the functional associated with (4.10) and $\bar{c}_{\widetilde{\Omega}_{\lambda}}$ is the corresponding mountain pass level;

(e) From (4.9),

$$
\liminf _{\lambda \rightarrow \infty} \int_{B_{l}(0) \cap \widetilde{\Omega}_{\lambda}}\left|v_{\lambda}\right|^{2} \geq \gamma
$$

Given $\rho>h>0$, we define

$$
D_{\rho, h} \doteq\left\{\left(x_{1}, \ldots, x_{N}\right) \in \mathbb{R}^{N} ; x_{N}>h\right\} \cap B_{\rho}(0) .
$$

From (1.7), $\chi_{\widetilde{\Omega}_{\lambda}} \rightarrow \chi_{\mathbb{R}_{+}^{N}}$ almost everywhere in $\mathbb{R}^{N}$, as $\lambda \rightarrow \infty$. Hence, $D_{\rho, h} \subset \widetilde{\Omega}_{\lambda}$ for every $\lambda$ sufficiently large. Thus, $v_{\lambda} \in H^{1}\left(D_{\rho, h}\right)$ for every $\lambda$ sufficiently large. By (a), we may assume that there exists $v_{\rho, h} \in H^{1}\left(D_{\rho, h}\right)$ such that

$$
\begin{cases}v_{\lambda} \rightarrow v_{\rho, h} & \text { weakly in } H^{1}\left(D_{\rho, h}\right), \text { as } \lambda \rightarrow \infty, \\ v_{\lambda} \rightarrow v_{\rho, h} & \text { strongly in } L^{p}\left(D_{\rho, h}\right), p \in\left[1,2^{*}\right), \text { as } \lambda \rightarrow \infty, \\ v_{\lambda}(x) \rightarrow v_{\rho, h}(x) & \text { a.e. in } D_{\rho, h}, \text { as } \lambda \rightarrow \infty .\end{cases}
$$

Using (a) one more time and the Banach-Steinhaus theorem, we find a constant $K>0$ such that

$$
\left\|v_{\rho, h}\right\|_{D_{\rho, h}} \leq K, \quad \text { for all } \rho, h>0
$$

(where $\|\cdot\|_{D_{\rho, h}}$ denotes the norm of $H^{1}\left(D_{\rho, h}\right)$ ). Let $\rho_{n} \rightarrow \infty$ and $h_{n} \rightarrow 0$ be monotone sequences. Thus,

$$
D_{n} \doteq D_{\rho_{n}, h_{n}} \subset D_{\rho_{n+1}, h_{n+1}} \doteq D_{n+1}, \quad \text { for all } n \geq 1 .
$$

This allows us to apply a diagonal type argument to obtain a bounded subsequence $\left(v_{k}\right)$ in $H^{1}\left(\mathbb{R}_{+}^{N}\right)$ and a function $v \in H^{1}\left(\mathbb{R}_{+}^{N}\right)$ such that

$$
\begin{cases}v_{k} \rightarrow v & \text { weakly in } H^{1}\left(\mathbb{R}_{+}^{N}\right), \text { as } k \rightarrow \infty, \\ v_{k} \rightarrow v & \text { strongly in } L_{\text {loc }}^{p}\left(\mathbb{R}_{+}^{N}\right), \text { for all } p \in\left[1,2^{*}\right), \text { as } k \rightarrow \infty, \\ v_{k}(x) \rightarrow v(x) & \text { a.e. in } \mathbb{R}_{+}^{N}, \text { as } k \rightarrow \infty .\end{cases}
$$


LEMMA 4.2. The function $v$ is a nontrivial weak solution of (3.1).

Proof. We first show that $v \not \equiv 0$. In fact, from (e),

$$
\liminf _{k \rightarrow \infty} \int_{B_{l}(0) \cap \widetilde{\Omega}_{k}} v_{k}^{2} \geq \gamma>0
$$

Given $t \in(0, l)$, define $A_{t}=\left\{x \in B_{l}(0) \cap \widetilde{\Omega}_{k} ; 0 \leq x^{N} \leq t\right\}$ and $\Lambda_{k}=\left(B_{l}(0) \cap\right.$ $\left.\widetilde{\Omega}_{k}\right) \backslash A_{t}$. Thus,

$$
\int_{B_{l}(0) \cap \widetilde{\Omega}_{k}} v_{k}^{2}=\left(\int_{A_{t}}+\int_{\Lambda_{k}}\right) v_{k}^{2} .
$$

As $\sup _{k}\left\|v_{k}\right\|_{D_{k}}<\infty$, using Hölder's inequality and the Sobolev embedding theorem, we get

$$
\int_{A_{t}} v_{k}^{2} \leq\left(\int_{A_{t}} v_{k}^{2^{*}}\right)^{2 / 2^{*}}\left(\int_{A_{t}} 1\right)^{2 / N} \leq \bar{K}\left|A_{t}\right|^{2 / N},
$$

for some constant $\bar{K}>0$. Now choose a $t \in(0, l)$ such that

$$
\int_{A_{t}} v_{k}^{2} \leq\left(\int_{A_{t}} v_{k}^{2^{*}}\right)^{2 / 2^{*}}\left(\int_{A_{t}} 1\right)^{2 / N} \leq \bar{K}\left|A_{t}\right|^{2 / N}<\frac{\gamma}{4} .
$$

Consequently, from (4.12), for all sufficiently large $k$, we have

$$
\frac{\gamma}{2} \leq \int_{B_{l}(0) \cap \widetilde{\Omega}_{k}} v_{k}^{2} \leq \frac{\gamma}{4}+\int_{\Lambda_{k}} v_{k}^{2} \leq \frac{\gamma}{4}+\int_{D} v_{k}^{2},
$$

for every compact set $D \subset \mathbb{R}^{N}$ with $\Lambda_{k} \subset D \subset D_{k}$. Hence, for all sufficiently large $k$,

$$
\int_{D} v_{k}^{2} \geq \frac{\gamma}{4}
$$

and consequently

$$
\int_{D} v^{2}=\lim _{k \rightarrow \infty} \int_{D} v_{k}^{2} \geq \frac{\gamma}{4}>0
$$

which implies $v \not \equiv 0$. In order to prove that $v$ is a weak solution of (3.1), we first show that $\nabla v_{k} \rightarrow \nabla v$, strongly in $\left(L^{2}(K)\right)^{N}$, for any compact set $K \subset \mathbb{R}_{+}^{N}$. Effectively, let $K \subset \mathbb{R}_{+}^{N}$ be a compact set. Taking $\psi \in C_{c}^{\infty}\left(\mathbb{R}_{+}^{N}\right)$ such that $\psi \equiv 1$, on $K$, and $0 \leq \psi \leq 1$, we have $\operatorname{supp} \psi \subset \widetilde{\Omega}_{k}$, for every $k$ sufficiently large. As $v_{k} \psi, v \psi \in H^{1}\left(\widetilde{\Omega}_{k}\right)$ and $v_{k}$ is a weak solution of (4.10), we have

$$
\begin{aligned}
\bar{J}_{\widetilde{\Omega}_{k}}^{\prime}\left(v_{k}\right)\left(v_{k} \psi\right) & =\int_{\widetilde{\Omega}_{k}}\left[\left|\nabla v_{k}\right|^{2} \psi+v_{k} \nabla v_{k} \nabla \psi+v_{k}^{2} \psi\right]-\int_{\widetilde{\Omega}_{k}} f\left(v_{k}^{2}\right) v_{k}^{2} \psi=0, \\
\bar{J}_{\widetilde{\Omega}_{k}}^{\prime}\left(v_{k}\right)(v \psi) & =\int_{\widetilde{\Omega}_{k}}\left[\psi \nabla v_{k} \nabla v+v \nabla v_{k} \nabla \psi+v_{k} v \psi\right]-\int_{\widetilde{\Omega}_{k}} f\left(v_{k}^{2}\right) v_{k} v \psi=0,
\end{aligned}
$$


where $\bar{J}_{\widetilde{\Omega}_{k}}: H^{1}\left(\widetilde{\Omega}_{k}\right) \rightarrow \mathbb{R}$ is the functional associated with (4.10). Combining (4.13)-(4.14), we obtain

$$
\begin{aligned}
& \int_{K}\left|\nabla v_{k}-\nabla v\right|^{2} \leq \int_{\mathbb{R}^{N}} \psi\left[\left|\nabla v_{k}\right|^{2}-2 \nabla v_{k} \nabla v+|\nabla v|^{2}\right] \\
&=\int_{\mathbb{R}_{+}^{N}}\left[\psi\left|\nabla v_{k}\right|^{2}-\psi \nabla v_{k} \nabla v+\psi \nabla v \nabla\left(v-v_{k}\right)\right] \\
&=\int_{\mathbb{R}_{+}^{N}}\left[f\left(v_{k}^{2}\right) v_{k}^{2} \psi-v_{k} \nabla v_{k} \nabla \psi-v_{k}^{2} \psi\right]+\int_{\mathbb{R}_{+}^{N}}\left[v \nabla v_{k} \nabla \psi+v_{k} v \psi\right] \\
&-\int_{\mathbb{R}_{+}^{N}} f\left(v_{k}^{2}\right) v_{k} v \psi+\int_{\mathbb{R}_{+}^{N}} \psi \nabla v \nabla\left(v-v_{k}\right) \\
&= \int_{\mathbb{R}_{+}^{N}}\left[f\left(v_{k}^{2}\right) \psi v_{k}\left(v_{k}-v\right)-\left(v_{k}-v\right) \nabla v_{k} \nabla \psi-v_{k} \psi\left(v_{k}-v\right)\right] \\
&+\int_{\mathbb{R}_{+}^{N}} \psi \nabla v \nabla\left(v_{k}-v\right) .
\end{aligned}
$$

This and the fact that $\left(v_{k}\right)$ is bounded in $L^{2}\left(\mathbb{R}_{+}^{N}\right)$ combined with $\left(\mathrm{f}_{6}\right),(4.11)$ and Hölder's inequality show that

$$
\int_{K}\left|\nabla v_{k}-\nabla v\right|^{2} \leq o_{k}(1), \quad \text { as } k \rightarrow \infty,
$$

that is $\nabla v_{k} \rightarrow \nabla v$, strongly in $\left(L^{2}(K)\right)^{N}$, as desired. As a consequence,

$$
\nabla v_{k}(x) \rightarrow \nabla v(x), \quad \text { for almost every } x \in \mathbb{R}^{N} \text {. }
$$

In order to conclude the proof of Lemma 4.2 , it remains to prove that

$$
\int_{\mathbb{R}_{+}^{N}}[\nabla v \nabla \phi+v \phi]-\int_{\mathbb{R}_{+}^{N}} f\left(v^{2}\right) v \phi=0, \quad \text { for all } \phi \in H^{1}\left(\mathbb{R}_{+}^{N}\right) .
$$

Since the set of restrictions of the functions of $C_{c}^{\infty}\left(\mathbb{R}^{N}\right)$ to $\mathbb{R}_{+}^{N}$ is a dense subspace of $H^{1}\left(\mathbb{R}_{+}^{N}\right)$ (see [10, Corollaire IX.8]), it suffices to show that relation (4.16) holds for every $\phi \in C_{c}^{\infty}\left(\mathbb{R}^{N}\right)$. Given $\phi \in C_{c}^{\infty}\left(\mathbb{R}^{N}\right)$, let $t>0$ be such that $B_{t}(0) \supset \operatorname{supp} \phi$. From (1.7), $\chi_{\widetilde{\Omega}_{k} \cap B_{t}(0)} \rightarrow \chi_{B_{t}^{+}}$almost everywhere in $\mathbb{R}^{N}$, as $k \rightarrow \infty$, where $B_{t}^{+} \doteq B_{t}(0) \cap \mathbb{R}_{+}^{N}$ and where $\chi_{B_{t}^{+}}$is the characteristic function related to the set $B_{t}^{+}$. This and (4.15) imply that $\chi_{\widetilde{\Omega}_{k} \cap B_{t}(0)} \nabla v_{k} \rightarrow \chi_{B_{t}^{+}} \nabla v$, almost everywhere in $\mathbb{R}^{N}$, as $k \rightarrow \infty$. Furthermore, $\left(\chi_{\widetilde{\Omega}_{k} \cap B_{t}(0)} \nabla v_{k}\right)_{k}$ is bounded in $\left(L^{2}\left(\mathbb{R}_{+}^{N}\right)\right)^{N}$. Hence, $\chi_{\widetilde{\Omega}_{k} \cap B_{t}(0)} \nabla v_{k}-\chi_{B_{t}^{+}} \nabla v$ weakly in $\left(L^{2}\left(\mathbb{R}_{+}^{N}\right)\right)^{N}$, as $k \rightarrow \infty$, and so

$$
\begin{aligned}
\lim _{k \rightarrow \infty} \int_{\widetilde{\Omega}_{k}} \nabla v_{k} \nabla \phi & =\lim _{k \rightarrow \infty} \int_{\mathbb{R}_{+}^{N}} \chi_{\widetilde{\Omega}_{k} \cap B_{t}(0)} \nabla v_{k} \nabla \phi \\
& =\int_{\mathbb{R}_{+}^{N}} \chi_{B_{t}^{+}} \nabla v \nabla \phi=\int_{\mathbb{R}_{+}^{N}} \nabla v \nabla \phi .
\end{aligned}
$$


Since $\left(v_{k}\right)$ is bounded in $H^{1}\left(\mathbb{R}_{+}^{N}\right)$, by $\left(\mathrm{f}_{6}\right)$, there exists $M_{1}>0$ such that

$$
\int_{B_{t}(0)}\left|f\left(v_{k}^{2}\right) v_{k}\right|^{q /(q-1)} \leq M_{1}
$$

Given $\eta>0$, from (4.11) and Egoroff's theorem, there exists $E \subset B_{t}(0)$ such that $|E|<\eta$ and $v_{k}(x) \rightarrow v(x)$ uniformly on $B_{t}(0) \backslash E$. Using Hölder's inequality, (4.18) and $\left(f_{6}\right)$, we get $M_{2}>0$ such that

$$
\left|\int_{B_{t}(0)}\left(f\left(v_{k}^{2}\right) v_{k}-f\left(v^{2}\right) v\right) \phi\right| \leq \int_{B_{t}(0) \backslash E}\left|f\left(v_{k}^{2}\right) v_{k}-f\left(v^{2}\right) v \| \phi\right|+M_{2} \eta^{q} .
$$

As $\eta>0$ can be chosen arbitrarily small, $f\left(v_{k}^{2}\right) v_{k} \rightarrow f\left(v^{2}\right) v$ uniformly on $B_{t}(0) \backslash$ $E$ and $\operatorname{supp} \phi \subset B_{t}(0)$, we obtain

$$
\lim _{k \rightarrow \infty} \int_{\widetilde{\Omega}_{k}} f\left(v_{k}^{2}\right) v_{k} \phi=\int_{\mathbb{R}_{+}^{N}} f\left(v^{2}\right) v \phi
$$

Using (4.11), similar arguments to those above show that

$$
\lim _{k \rightarrow \infty} \int_{\widetilde{\Omega}_{k}} v_{k} \phi=\int_{\mathbb{R}_{+}^{N}} v \phi
$$

Combing (4.17)-(4.20) with the fact that $v_{k}$ satisfies (4.10), yields

$$
0=\lim _{k \rightarrow \infty} \int_{\widetilde{\Omega}_{k}}\left(\nabla v_{k} \nabla \phi+v_{k} \phi-f\left(v_{k}^{2}\right) v_{k} \phi\right)=\int_{\mathbb{R}_{+}^{N}}\left(\nabla v \nabla \phi+v \phi-f\left(v^{2}\right) v \phi\right),
$$

for every $\phi \in C_{c}^{\infty}\left(\mathbb{R}^{N}\right)$, and the proof Lemma 4.2 is complete.

In the following, we conclude the proof of Propostion 4.1. From (4.1) and (4.4),

$$
\begin{aligned}
c_{\infty}+o_{k}(1) & \geq \bar{c}_{\Omega_{k}}=\bar{c}_{\widetilde{\Omega}_{k}}=\bar{J}_{\widetilde{\Omega}_{k}}\left(v_{k}\right) \\
& =\bar{J}_{\widetilde{\Omega}_{k}}\left(v_{k}\right)-\frac{1}{2} \bar{J}_{\widetilde{\Omega}_{k}}^{\prime}\left(v_{k}\right) v_{k}=\frac{1}{2} \int_{\widetilde{\Omega}_{k}}\left[f\left(v_{k}^{2}\right) v_{k}^{2}-F\left(v_{k}^{2}\right)\right] .
\end{aligned}
$$

Using Fatou's lemma and (4.11), we have

$$
\begin{aligned}
c_{\infty} & \geq \limsup _{k \rightarrow \infty} \bar{c}_{\widetilde{\Omega}_{k}} \geq \liminf _{k \rightarrow \infty} \bar{c}_{\widetilde{\Omega}_{k}}=\liminf _{k \rightarrow \infty} \frac{1}{2} \int_{\widetilde{\Omega}_{k}}\left[f\left(v_{k}^{2}\right) v_{k}^{2}-F\left(v_{k}^{2}\right)\right] \\
& \geq \frac{1}{2} \int_{\mathbb{R}_{+}^{N}}\left[f\left(v^{2}\right) v^{2}-F\left(v^{2}\right)\right]=J_{\infty}(v) \geq c_{\infty} .
\end{aligned}
$$

Consequently, $\lim _{\lambda \rightarrow \infty} \bar{c}_{\Omega_{\lambda}}=c_{\infty}$, that is, (4.5) holds, and the proof of Proposition 4.1 is complete. 


\section{The barycenter map}

This section is devoted to establish a key relation between some subsets of $\mathbb{R}^{N}$ and $M_{\lambda}$. For $q \in\left(2,2^{*}\right)$ given by $\left(\mathrm{f}_{5}\right)$ and $\lambda>0$, define the barycenter $\operatorname{map} \beta_{\lambda}: M_{\lambda} \rightarrow \mathbb{R}^{N}$ by

$$
\beta_{\lambda}(u)=\frac{\int_{\Omega_{\lambda}} x|u|^{q} d x}{\int_{\Omega_{\lambda}}|u|^{q} d x} .
$$

Proposition 5.1. Let $\left(\Gamma_{1}^{+}\right)_{\lambda}$ be the expanding set $\lambda \Gamma_{1}^{+}$. Then, there exist $\varepsilon^{*}>0$ and $\lambda_{1}>0$ such that $\beta_{\lambda}(u) \in\left(\Gamma_{1}^{+}\right)_{\lambda}$, provided that $\lambda>\lambda_{1}, u \in M_{\lambda}$ and $I_{\lambda}(u) \leq b_{\lambda}^{*}$, where $b_{\lambda}^{*}=b_{\lambda}+\varepsilon^{*}$.

Proof. It suffices to show that if $\left(\varepsilon_{n}\right)$ and $\left(\lambda_{n}\right)$ are arbitrary sequences, with $\varepsilon_{n} \rightarrow 0$ and $\lambda_{n} \rightarrow \infty$, and if $u_{n} \in M_{\lambda_{n}}$ is a sequence such that

$$
b_{\lambda_{n}} \leq I_{\lambda_{n}}\left(u_{n}\right) \leq b_{\lambda_{n}}+\varepsilon_{n},
$$

then

$$
\operatorname{dist}\left(\beta_{\lambda_{n}}\left(u_{n}\right), \Gamma_{1 \lambda_{n}}\right) \leq \lambda_{n} r
$$

for every $n$ sufficiently large. In fact, by (5.1) and Proposition 4.1,

$$
I_{\lambda_{n}}\left(u_{n}\right) \rightarrow c_{\infty}, \quad \text { as } n \rightarrow \infty .
$$

Using that $u_{n} \in M_{\lambda_{n}}$ and (1.6), there exists $t_{n}>0$ such that

$$
\begin{aligned}
o_{n}(1)+c_{\infty} & =I_{\lambda_{n}}\left(u_{n}\right) \geq \max _{t \geq 0} I_{\lambda_{n}}\left(t u_{n}\right) \\
& \geq \max _{t \geq 0} J_{\lambda_{n}}\left(t\left|u_{n}\right|\right)=J_{\lambda_{n}}\left(t_{n}\left|u_{n}\right|\right) \geq c_{\lambda_{n}},
\end{aligned}
$$

where $J_{\lambda_{n}}$ is the functional associated with (4.2) with $\lambda=\lambda_{n}, c_{\lambda_{n}}$ and $N_{\lambda_{n}}$ are the corresponding mountain pass level and the Nehari manifold. Combining (4.4)-(4.5) with (5.4) and Proposition 4.1, we have

$$
\lim _{n \rightarrow \infty} c_{\lambda_{n}}=\lim _{n \rightarrow \infty} J_{\lambda_{n}}\left(t_{n}\left|u_{n}\right|\right)=c_{\infty} .
$$

Set $\bar{\varepsilon}_{n}=b_{\lambda_{n}}-c_{\lambda_{n}}$. By Proposition 4.1 and (5.5), $\bar{\varepsilon}_{n} \rightarrow 0$, as $n \rightarrow \infty$. Thus,

$$
\bar{\varepsilon}_{n}+c_{\lambda_{n}} \geq J_{\lambda_{n}}\left(t_{n}\left|u_{n}\right|\right) \geq c_{\lambda_{n}} .
$$

Applying the variational principle [21, Corollary 3.4], for every $n \in \mathbb{N}$, there exists $v_{n} \in N_{\lambda_{n}}$ such that

$$
\left\|t_{n}\left|u_{n}\right|-v_{n}\right\|_{\Omega_{n}} \leq 2 \sqrt{\bar{\varepsilon}_{n}}, \quad c_{\lambda_{n}} \leq J_{\lambda_{n}}\left(v_{n}\right) \leq c_{\lambda_{n}}+2 \bar{\varepsilon}_{n}
$$

and

$$
\left\|\left(\left.J_{\lambda_{n}}\right|_{N_{\lambda_{n}}}\right)^{\prime}\left(v_{n}\right)\right\|_{\left(H^{1}\left(\Omega_{\lambda_{n}}, \Gamma_{0 \lambda_{n}}\right)\right)^{\prime}} \leq 8 \sqrt{\bar{\varepsilon}_{n}} .
$$


As in the proof of Proposition 2.3, we find that $v_{n} \in H^{1}\left(\Omega_{\lambda_{n}}, \Gamma_{0 \lambda_{n}}\right)$ satisfies

$$
J_{\lambda_{n}}\left(v_{n}\right) \rightarrow c_{\infty}, \quad J_{\lambda_{n}}^{\prime}\left(v_{n}\right) \rightarrow 0 .
$$

From (5.7) and $\left(\mathrm{f}_{3}\right)$, the sequence $\left(\left\|v_{n}\right\|_{\Omega_{\lambda_{n}}}\right)_{n}$ is bounded. Consequently, from Lemma 3.1 and (5.7), there exist $l>0, \gamma>0$ and $y_{n} \in \mathbb{R}^{N}$ such that

$$
\liminf _{n \rightarrow \infty} \int_{B_{l}\left(y_{n}\right) \cap \Omega_{\lambda_{n}}}\left|v_{n}\right|^{2} \geq \gamma>0 .
$$

Proceeding as in the proof of Lemma 4.1, with (5.7) replacing (4.6), we get a positive constant $C>0$ such that $\operatorname{dist}\left(y_{n}, \partial \Omega_{\lambda_{n}}\right) \leq C$. Thus, by increasing $l$ if necessary, we may assume that $y_{n} \in \partial \Omega_{\lambda_{n}}$. Following the same argument in the proof of Proposition 4.1, we define

$$
\widetilde{v}_{n}(x)=v_{n}\left(T_{y_{n}}^{-1} x+y_{n}\right), \quad \text { for all } x \in \widetilde{\Omega}_{n} \doteq T_{y_{n}}\left(\Omega_{\lambda_{n}}-\left\{y_{n}\right\}\right) \text {, for all } n \in \mathbb{N} \text {, }
$$

to obtain a subsequence of $\widetilde{v}_{n} \in H^{1}\left(\widetilde{\Omega}_{n}, \widetilde{\Gamma}_{0 \lambda_{n}}\right)$ (still denoted by $\widetilde{v}_{n}$ ) and a function $v \in H^{1}\left(\mathbb{R}_{+}^{N}\right)$ such that

$(5.8) \quad \widetilde{v}_{n} \rightarrow v \quad$ in $H^{1}\left(\mathbb{R}_{+}^{N}\right), \quad \widetilde{v}_{n} \rightarrow v \quad$ in $L_{\text {loc }}^{p}\left(\mathbb{R}_{+}^{N}\right), \quad$ for all $\mathrm{p} \in\left[1,2^{*}\right)$,

$$
\widetilde{v}_{n}(x) \rightarrow v(x), \quad \nabla \widetilde{v}_{n}(x) \rightarrow \nabla v(x) \quad \text { for almost every } x \in \mathbb{R}_{+}^{N} .
$$

Claim 1. There exits a constant $C>0$ such that $\operatorname{dist}\left(y_{n}, \Gamma_{1 \lambda_{n}}\right) \leq C$.

In fact, suppose Claim I were false. Then we could find subsequences (not renamed) such that

$$
\alpha_{n} \doteq \operatorname{dist}\left(y_{n}, \Gamma_{1 \lambda_{n}}\right) \rightarrow \infty, \quad \text { as } n \rightarrow \infty \text {. }
$$

We next show that $v \in H^{1}\left(\mathbb{R}_{+}^{N}\right)$ is a weak solution of

$$
-\Delta v+v=f\left(v^{2}\right) v \quad \text { in } \mathbb{R}_{+}^{N}, \quad v=0 \quad \text { on } \mathbb{R}^{N-1} .
$$

Effectively, set

$$
w_{n}(x) \doteq \xi\left(\frac{|x|}{\alpha_{n}}\right) \widetilde{v}_{n}(x), \quad \text { for all } x \in \widetilde{\Omega}_{n}
$$

where $\alpha_{n}>0$ is given in (5.10) and $\xi \in C_{c}^{\infty}\left(\mathbb{R}_{+}\right)$is such that $\xi(t)=1, t \in$ $[0,1 / 2], \xi(t)=0, t \geq 2 / 3$. Thus, $w_{n} \in H_{0}^{1}\left(\widetilde{\Omega}_{n}\right) \subset H_{0}^{1}\left(\mathbb{R}_{+}^{N}\right)$ and $w_{n}(x) \rightarrow v(x)$ almost every $x \in \mathbb{R}_{+}^{N}$, as $n \rightarrow \infty$. Since $\left(w_{n}\right) \subset H_{0}^{1}\left(\mathbb{R}_{+}^{N}\right)$ is bounded, there is $w \in H_{0}^{1}\left(\mathbb{R}_{+}^{N}\right)$ such that $w_{n} \rightarrow w$ weakly in $H_{0}^{1}\left(\mathbb{R}_{+}^{N}\right)$. By the Sobolev imbedding theorem, $w_{n}(x) \rightarrow w(x)$ almost every $x \in \mathbb{R}_{+}^{N}$, as $n \rightarrow \infty$. As the limit is unique, $v \equiv w$ in $H_{0}^{1}\left(\mathbb{R}_{+}^{N}\right)$. Taking $\phi \in C_{c}^{\infty}\left(\mathbb{R}_{+}^{N}\right)$, gives supp $\phi \subset \widetilde{\Omega}_{n}$ for every $n$ sufficiently large. By (5.7) and the definition of $\widetilde{v}_{n}$, we have

$$
\int_{\widetilde{\Omega}_{n}}\left(\nabla \widetilde{v}_{n} \nabla \phi+\widetilde{v}_{n} \phi-f\left(\widetilde{v}_{n}^{2}\right) \widetilde{v}_{n} \phi\right)=o_{1}(n),
$$


for every $n$ sufficiently large. From (5.8), after taking $n \rightarrow \infty$ in (5.12), we find

$$
\int_{\mathbb{R}_{+}^{N}}\left(\nabla v \nabla \phi+v \phi-f\left(v^{2}\right) v \phi\right)=0 .
$$

Since $\phi$ is arbitrary, the function $v$ is a weak solution of (5.11).

Let $J_{\widetilde{\Omega}_{n}}: H^{1}\left(\widetilde{\Omega}_{n}, \widetilde{\Gamma}_{\lambda_{n} 0}\right) \rightarrow \mathbb{R}$ be the functional associated with the problem

$$
\begin{cases}-\Delta v+v=f\left(v^{2}\right) v & \text { in } \widetilde{\Omega}_{n}, \\ \frac{\partial v}{\partial \nu}=0 & \text { on } \widetilde{\Gamma}_{1 \lambda_{n}}, \\ v=0 & \text { on } \widetilde{\Gamma}_{0 \lambda_{n}} .\end{cases}
$$

Using that $v$ is a weak solution of (5.11), Fatou lemma and (5.7), we have

$$
\begin{aligned}
c_{\infty} & =\liminf _{n \rightarrow \infty} J_{\widetilde{\Omega}_{n}}\left(\widetilde{v}_{n}\right)=\liminf _{n \rightarrow \infty} \frac{1}{2} \int_{\widetilde{\Omega}_{n}}\left(f\left(\widetilde{v}_{n}^{2}\right) \widetilde{v}_{n}^{2}-F\left(\widetilde{v}_{n}^{2}\right)\right) \\
& =\liminf _{n \rightarrow \infty} \frac{1}{2} \int_{\mathbb{R}_{+}^{N}} \chi_{\widetilde{\Omega}_{n}}\left(f\left(\widetilde{v}_{n}^{2}\right) \widetilde{v}_{n}^{2}-F\left(\widetilde{v}_{n}^{2}\right)\right) \\
& \geq \frac{1}{2} \int_{\mathbb{R}_{+}^{N}}\left(f\left(v^{2}\right) v^{2}-F\left(v^{2}\right)\right) \geq c_{\mathbb{R}_{+}^{N}} \geq c_{\infty},
\end{aligned}
$$

that is $c_{\infty}=c_{\mathbb{R}_{+}^{N}}$. However, $c_{\infty}=c_{\mathbb{R}_{+}^{N}} \geq c_{\mathbb{R}^{N}}=2 c_{\infty}$, which is impossible, and Claim I is proved.

Claim II. Given any $\varepsilon>0$, there exists $R=R(\varepsilon)>0$ such that

$$
\lim _{n \rightarrow \infty} \int_{\Omega_{\lambda_{n}} \cap B_{R}\left(y_{n}\right)}\left[f\left(v_{n}^{2}\right) v_{n}^{2}-F\left(v_{n}^{2}\right)\right] \geq c_{\infty}-\varepsilon .
$$

Indeed, we first show that the function $v$ given by (5.8)-(5.9) satisfies $J_{\infty}(v)=$ $c_{\infty}$ and $v$ is a solution of (3.1). Consider $\phi \in C_{0}^{\infty}\left(\mathbb{R}^{N}\right)$ such that $\phi=1$, on $B_{1}(0)$, $\phi=0$, on $B_{2}^{c}(0), 0 \leq \phi \leq 1$, and define

$$
\phi_{T}(x)=\phi\left(\frac{x}{T}\right), \quad \text { for all } x \in \mathbb{R}^{N}, T>0 .
$$

Hence, the sequence $\phi_{T} \widetilde{v}_{n}$ is bounded in $H^{1}\left(\widetilde{\Omega}_{n}, \widetilde{\Gamma}_{0 \lambda_{n}}\right)$ and $\phi_{T} v \rightarrow v$ in $H^{1}\left(\mathbb{R}_{+}^{N}\right)$, as $T \rightarrow \infty$. By (5.7), we have

$$
\int_{\widetilde{\Omega}_{n}} \nabla \widetilde{v}_{n} \nabla\left(\phi_{T} \widetilde{v}_{n}\right)+\int_{\widetilde{\Omega}_{n}}\left|\widetilde{v}_{n}\right|^{2} \phi_{T}=\int_{\widetilde{\Omega}_{n}} f\left(\widetilde{v}_{n}^{2}\right) \widetilde{v}_{n}^{2} \phi_{T}+o_{n}(1),
$$

that is,

$$
\int_{\widetilde{\Omega}_{n}}\left|\nabla \widetilde{v}_{n}\right|^{2} \phi_{T}+\int_{\widetilde{\Omega}_{n}} \widetilde{v}_{n} \nabla \widetilde{v}_{n} \nabla \phi_{T}+\int_{\widetilde{\Omega}_{n}}\left|\widetilde{v}_{n}\right|^{2} \phi_{T}=\int_{\widetilde{\Omega}_{n}} f\left(\widetilde{v}_{n}^{2}\right) \widetilde{v}_{n}^{2} \phi_{T}+o_{n}(1) .
$$


Mixed Boundary Problem for Schrödinger Equations

353

We now proceed to verify that

$$
\begin{aligned}
\int_{\widetilde{\Omega}_{n}} \widetilde{v}_{n} \nabla \widetilde{v}_{n} \nabla \phi_{T} & \rightarrow \int_{\mathbb{R}_{+}^{N}} v \nabla v \nabla \phi_{T}, \\
\int_{\widetilde{\Omega}_{n}}\left|\widetilde{v}_{n}\right|^{2} \phi_{T} & \rightarrow \int_{\mathbb{R}_{+}^{N}}|v|^{2} \phi_{T}, \\
\int_{\widetilde{\Omega}_{n}} f\left(\widetilde{v}_{n}^{2}\right) \widetilde{v}_{n}^{2} \phi_{T} & \rightarrow \int_{\mathbb{R}_{+}^{N}} f\left(v^{2}\right) v^{2} \phi_{T},
\end{aligned}
$$

as $n \rightarrow \infty$. Let $\varepsilon>0$ and $T>1$ be arbitrary numbers. Fix $t>0$ to be appropriately chosen and define

$$
E_{t} \doteq\left\{x \in B_{2 T}(0) ; 0 \leq x^{N} \leq t\right\}
$$

Using that $\left(\left\|\widetilde{v}_{n}\right\|_{\widetilde{\Omega}_{n}}\right)_{n}$ is bounded and Hölder inequality, we obtain

$$
\begin{aligned}
& \int_{E_{t}}\left|\widetilde{v}_{n}\right|^{2} \leq\left(\int_{E_{t}}\left|\widetilde{v}_{n}\right|^{2^{*}}\right)^{2 / 2^{*}}\left(\int_{E_{t}}|1|^{N}\right)^{2 / N} \leq M\left|E_{t}\right|^{2 / N} \leq M T^{2(N-1) / N} t^{2 / N}, \\
& \int_{E_{t}}\left|\widetilde{v}_{n}\right|^{q} \leq\left(\int_{E_{t}}\left|\widetilde{v}_{n}\right|^{2^{*}}\right)^{q / 2^{*}}\left(\int_{E_{t}}|1|^{\alpha}\right)^{q / \alpha} \leq M\left|E_{t}\right|^{2 / N} \leq M T^{(N-1) q / \alpha} t^{q / \alpha},
\end{aligned}
$$

for some positive constant $M$, where $\alpha=2^{*} /\left(2^{*}-q\right)$. Set $\kappa \doteq \max \{\alpha / q, N / 2\}$ and take $t \doteq \varepsilon^{\kappa} T^{1-N}$. Thus, $T^{(N-1) 2 / N} t^{2 / N}=\varepsilon^{2 \kappa / N}$ and $T^{(N-1) q / \alpha} t^{q / \alpha}=$ $\varepsilon^{\kappa q / \alpha}$, with $\min \{2 \kappa / N, \kappa q / \alpha\}=1>0$, and consequently

$$
\lim _{\varepsilon \rightarrow 0} \varepsilon^{\kappa q / \alpha}=\lim _{\varepsilon \rightarrow 0} \varepsilon^{2 \kappa / N}=0 .
$$

By choice of $t$, we have

$$
\int_{E_{t}}\left|\widetilde{v}_{n}\right|^{2} \leq \varepsilon^{2 \kappa / N} M \quad \text { and } \quad \int_{E_{t}}\left|\widetilde{v}_{n}\right|^{q} \leq \varepsilon^{\kappa q / \alpha} M .
$$

We observe that by (5.8)-(5.9), $v$ also satisfies (5.20). Furthermore, $B_{2 T} \backslash E_{t} \subset$ $\widetilde{\Omega}_{n}$, provided that $n$ is sufficiently large. Applying Hölder inequality, (5.8), (5.9) and (5.20), for every $n$ sufficiently large, we get

$$
\begin{aligned}
& \left|\int_{\widetilde{\Omega}_{n}} \widetilde{v}_{n} \nabla \phi_{T} \nabla \widetilde{v}_{n}-\int_{\mathbb{R}_{+}^{N}} v \nabla \phi_{T} \nabla v\right| \\
& \leq\left|\int_{B_{2 T} \backslash E_{t}} \widetilde{v}_{n} \nabla \phi_{T} \nabla \widetilde{v}_{n}-v \nabla \phi_{T} \nabla v\right|+\left|\int_{E_{t}} \widetilde{v}_{n} \nabla \phi_{T} \nabla \widetilde{v}_{n}\right|+\left|\int_{E_{t}} v \nabla \phi_{T} \nabla v\right| \\
& \quad \leq\left|\int_{B_{2 T} \backslash E_{t}} \nabla \phi_{T} \nabla \widetilde{v}_{n}\left(\widetilde{v}_{n}-v\right)\right|+\left|\int_{B_{2 T} \backslash E_{t}} v \nabla \phi_{T}\left(\nabla \widetilde{v}_{n}-\nabla v\right)\right| \\
& \quad+M \int_{E_{t}}\left(\left|\widetilde{v}_{n}\right|^{2}+|v|^{2}\right) \leq o_{n}(1)+2 M \varepsilon^{2 \kappa / N} .
\end{aligned}
$$


From (5.19) and the fact that $\varepsilon$ can be chosen arbitrarily small, we obtain that (5.16) holds for every $T>0$. We can proceed analogously to proof of (5.17). In order to verify $(5.18)$, we combine $\left(\mathrm{f}_{6}\right)$ with $(5.20)$, to obtain

$$
\int_{E_{t}} f\left(\widetilde{v}_{n}^{2}\right) \widetilde{v}_{n}^{2} \leq \varepsilon \int_{E_{t}} \widetilde{v}_{n}^{2}+C_{\varepsilon} \int_{E_{t}} \widetilde{v}_{n}^{q} \leq M\left(\varepsilon^{\kappa q / \alpha}+\varepsilon^{2 \kappa / N}\right) .
$$

From (5.8) and (5.21), we have

$$
\begin{aligned}
& \left|\int_{\widetilde{\Omega}_{n}} f\left(\widetilde{v}_{n}^{2}\right) \widetilde{v}_{n}^{2} \phi_{T}-\int_{\mathbb{R}_{+}^{N}} f\left(v^{2}\right) v^{2} \phi_{T}\right| \\
& \leq\left|\int_{B_{2 T} \backslash E_{t}}\left(f\left(\widetilde{v}_{n}^{2}\right) \widetilde{v}_{n}^{2}-f\left(v^{2}\right) v^{2}\right) \phi_{T}\right|+\left|\int_{E_{t}} f\left(\widetilde{v}_{n}^{2}\right) \widetilde{v}_{n}^{2}\right|+\left|\int_{E_{t}} f\left(v^{2}\right) v^{2}\right| \\
& \leq o_{n}(1)+M\left(\varepsilon^{\kappa q / \alpha}+\varepsilon^{2 \kappa / N}\right) .
\end{aligned}
$$

From (5.19) and the fact that $\varepsilon$ can be chosen arbitrarily small, we obtain that (5.18) holds for every $T>0$. Combining (5.15)-(5.18) with Fatou lemma, we get

$$
\int_{\mathbb{R}_{+}^{N}}|\nabla v|^{2} \phi_{T}+\int_{\mathbb{R}_{+}^{N}} v \nabla v \nabla \phi_{T}+\int_{\mathbb{R}_{+}^{N}}|v|^{2} \phi_{T} \leq \int_{\mathbb{R}_{+}^{N}} f\left(v^{2}\right) v^{2} \phi_{T},
$$

for every $T>0$. Finally, taking $T \rightarrow+\infty$, we find

$$
\int_{\mathbb{R}_{+}^{N}}\left[|\nabla v|^{2}+v^{2}\right] \leq \int_{\mathbb{R}_{+}^{N}} f\left(v^{2}\right) v^{2} .
$$

From $\left(\mathrm{f}_{1}\right)-\left(\mathrm{f}_{4}\right)$, there exists $t_{0}>0$ such that $t_{0} v \in N_{\infty}$. By (5.22), we have $0<t_{0} \leq 1$. Suppose that $t_{0}<1$. In this case, using that the function $s \rightarrow$ $f(s) s-F(s)$ is increasing in $[0,+\infty)$, by $\left(\mathrm{f}_{4}\right)$, Fatou lemma and (5.9), we have

$$
\begin{aligned}
c_{\infty} & =\liminf _{n \rightarrow \infty}\left[J_{\widetilde{\Omega}_{n}}\left(\widetilde{v}_{n}\right)-\frac{1}{2} J_{\widetilde{\Omega}_{n}}^{\prime}\left(\widetilde{v}_{n}\right) \widetilde{v}_{n}\right] \\
& =\liminf _{n \rightarrow \infty} \frac{1}{2} \int_{\widetilde{\Omega}_{n}}\left[f\left(\widetilde{v}_{n}^{2}\right) \widetilde{v}_{n}^{2}-F\left(\widetilde{v}_{n}^{2}\right)\right] \geq \frac{1}{2} \int_{\mathbb{R}_{+}^{N}}\left[f\left(v^{2}\right) v^{2}-F\left(v^{2}\right)\right] \\
& >\frac{1}{2} \int_{\mathbb{R}_{+}^{N}}\left[f\left(t_{0}^{2} v^{2}\right) t_{0}^{2} v^{2}-F\left(t_{0}^{2} v^{2}\right)\right]=J_{\infty}\left(t_{0} v\right)-\frac{1}{2} J_{\infty}^{\prime}\left(t_{0} v\right) t_{0} v \geq c_{\infty},
\end{aligned}
$$

which is impossible. Hence, $t_{0}=1$, and consequently $v \in N_{\infty}$. Furthermore, $v$ satisfies

$$
c_{\infty} \geq \frac{1}{2} \int_{\mathbb{R}_{+}^{N}}\left[f\left(v^{2}\right) v^{2}-F\left(v^{2}\right)\right]=J_{\infty}(v)-\frac{1}{2} J_{\infty}^{\prime}(v) v=J_{\infty}(v) \geq c_{\infty} .
$$

We conclude that $J_{\infty}(v)=c_{\infty}$ and $v$ is a solution of (3.1). By (5.23), given any $\varepsilon>0$, there exists $R>0$ such that

$$
\frac{1}{2} \int_{\mathbb{R}_{+}^{N} \cap B_{R}(0)}\left[f\left(v^{2}\right) v^{2}-F\left(v^{2}\right)\right] \geq c_{\infty}-\varepsilon
$$


Since $\chi_{B_{R} \cap \widetilde{\Omega}_{n}}(x) \widetilde{v}_{n}(x) \rightarrow \chi_{B_{R}^{+}}(x) v(x)$ almost every $x \in \mathbb{R}_{+}^{N}$, as $n \rightarrow \infty$, by Fatou lemma we have

$$
\begin{array}{r}
\liminf _{n \rightarrow \infty} \frac{1}{2} \int_{B_{R}\left(y_{n}\right) \cap \Omega_{n}}\left[f\left(v_{n}^{2}\right) v_{n}^{2}-F\left(v_{n}^{2}\right)\right]=\liminf _{n \rightarrow \infty} \frac{1}{2} \int_{B_{R} \cap \widetilde{\Omega}_{n}}\left[f\left(\widetilde{v}_{n}^{2}\right) \widetilde{v}_{n}^{2}-F\left(\widetilde{v}_{n}^{2}\right)\right] \\
\geq \frac{1}{2} \int_{\mathbb{R}_{+}^{N} \cap B_{R}(0)}\left[f\left(v^{2}\right) v^{2}-F\left(v^{2}\right)\right] \geq c_{\infty}-\varepsilon
\end{array}
$$

which completes the proof of Claim II.

We are now ready to show (5.2). By (5.6) and the Sobolev embedding theorem, the sequences $\left\{t_{n}\left|u_{n}\right|\right\} \subset H^{1}\left(\Omega_{n}, \Gamma_{0 \lambda_{n}}\right)$ and $\left\{v_{n}\right\} \subset H^{1}\left(\Omega_{n}, \Gamma_{0 \lambda_{n}}\right)$ have the same limit. Hence, Claim II is also valid for $\left\{t_{n}\left|u_{n}\right|\right\}_{n}$, that is,

$$
\liminf _{n \rightarrow \infty} \frac{1}{2} \int_{B_{R}\left(y_{n}\right) \cap \Omega_{\lambda_{n}}}\left[f\left(\left|t_{n} u_{n}\right|^{2}\right)\left|t_{n} u_{n}\right|^{2}-F\left(\left|t_{n} u_{n}\right|^{2}\right)\right] \geq c_{\infty}-\varepsilon .
$$

From this, (5.5) and $\left(f_{5}\right)$, we have

$$
\liminf _{n \rightarrow \infty} \int_{\Omega_{\lambda_{n}} \backslash B_{R}\left(y_{n}\right)} C\left|t_{n} u_{n}\right|^{q} \leq \varepsilon .
$$

By Claim I, we can assume that $y_{n} \in \Gamma_{1 \lambda_{n}}$, i.e. $y_{n} / \lambda_{n} \in \Gamma_{1}$ and $y_{n} / \lambda_{n} \rightarrow x_{0} \in \bar{\Gamma}_{1}$, as $n \rightarrow \infty$, because $\bar{\Gamma}_{1}$ is a compact set. Take $j \in\{1, \ldots, N\}$. From the definition of the barycenter, we have

$$
\left|\frac{\beta_{\lambda_{n}}^{j}\left(u_{n}\right)}{\lambda_{n}}-x_{0}^{j}\right| \leq \frac{\int_{\Omega_{\lambda_{n}}}\left|\frac{x^{j}}{\lambda_{n}}-x_{0}^{j}\right|\left|t_{n} u_{n}\right|^{q}}{\int_{\Omega_{\lambda_{n}}}\left|t_{n} u_{n}\right|^{q}} .
$$

Using Lemma 2.1 and the fact that $t_{n}\left|u_{n}\right| \in M_{\lambda_{n}}$, we may assume that

$$
\int_{\Omega_{\lambda_{n}}}\left|t_{n} u_{n}\right|^{q} \geq \gamma>0, \quad \text { for all } n \in \mathbb{N} .
$$

As a consequence,

$$
\begin{aligned}
& \gamma\left|\frac{\beta_{\lambda_{n}}^{j}\left(u_{n}\right)}{\lambda_{n}}-x_{0}^{j}\right| \leq \int_{\Omega_{\lambda_{n}}}\left|\frac{x^{j}}{\lambda_{n}}-x_{0}^{j}\right|\left|t_{n} u_{n}\right|^{q} \\
& =\int_{\Omega_{\lambda_{n}} \cap B_{R}\left(y_{n}\right)}\left|\frac{x^{j}}{\lambda_{n}}-x_{0}^{j}\right|\left|t_{n} u_{n}\right|^{q}+\int_{\Omega_{\lambda_{n}} \backslash B_{R}\left(y_{n}\right)}\left|\frac{x^{j}}{\lambda_{n}}-x_{0}^{j}\right|\left|t_{n} u_{n}\right|^{q} \\
& \leq \int_{\Omega_{\lambda_{n}} \cap B_{R}\left(y_{n}\right)}\left|\frac{x^{j}}{\lambda_{n}}-\frac{y_{n}^{j}}{\lambda_{n}}\right|\left|t_{n} u_{n}\right|^{q}+\int_{\Omega_{\lambda_{n}} \cap B_{R}\left(y_{n}\right)}\left|\frac{y_{n}^{j}}{\lambda_{n}}-x_{0}^{j}\right|\left|t_{n} u_{n}\right|^{q} \\
& +\int_{\Omega_{\lambda_{n}} \backslash B_{R}\left(y_{n}\right)}\left|\frac{x^{j}}{\lambda_{n}}-x_{0}^{j}\right|\left|t_{n} u_{n}\right|^{q}
\end{aligned}
$$




$$
\begin{aligned}
& \leq \frac{R}{\lambda_{n}} \int_{\Omega_{\lambda_{n}}}\left|t_{n} u_{n}\right|^{q}+\left|\frac{y_{n}}{\lambda_{n}}-x_{0}\right| \int_{\Omega_{\lambda_{n}}}\left|t_{n} u_{n}\right|^{q}+\operatorname{diam}(\Omega) \int_{\Omega_{\lambda_{n}} \backslash B_{R}\left(y_{n}\right)}\left|t_{n} u_{n}\right|^{q} \\
& =\left(\frac{R}{\lambda_{n}}+\left|\frac{y_{n}}{\lambda_{n}}-x_{0}\right|\right) \int_{\Omega_{n}}\left|t_{n} u_{n}\right|^{q}+\operatorname{diam}(\Omega) \int_{\Omega_{\lambda_{n}} \backslash B_{R}\left(y_{n}\right)}\left|t_{n} u_{n}\right|^{q} .
\end{aligned}
$$

From (5.24) and the fact that the sequence $\left(\left\|t_{n} u_{n}\right\|_{A_{\lambda_{n}}}\right)_{n}$ is bounded and $y_{n} / \lambda_{n} \rightarrow$ $x_{0}$, we find

$$
0 \leq \liminf _{n \rightarrow \infty}\left|\frac{\beta_{\lambda_{n}}^{j}\left(u_{n}\right)}{\lambda_{n}}-x_{0}^{j}\right| \leq \operatorname{diam}(\Omega) \frac{\varepsilon}{\gamma C}, \quad \text { for all } j \in\{1, \ldots, N\} .
$$

Since $\varepsilon>0$ is arbitrary, we can find a subsequence (not renamed) such that

$$
\operatorname{dist}\left(\frac{\beta_{\lambda_{n}}\left(u_{n}\right)}{\lambda_{n}}, \Gamma_{1}\right) \rightarrow 0, \quad \text { as } n \rightarrow \infty .
$$

We conclude that $\operatorname{dist}\left(\beta_{\lambda_{n}}\left(u_{n}\right), \Gamma_{1 \lambda_{n}}\right) \leq \lambda_{n} r$, for every $n$ sufficiently large, hence that (5.2) holds, and Proposition 5.1 follows.

Taking $\varepsilon^{*}>0$ given by Proposition 5.1, we define $b_{\lambda}^{*}=b_{\lambda}+\varepsilon^{*}$. As a consequence of Propositions 3.1, 4.1 and 5.1, we obtain the following result which is the key point in the comparison of the topology of the sublevel sets of the functional $I_{\lambda}$ with that of $\Gamma_{1 \lambda}$.

Lemma 5.1. There exists $\lambda^{*}>0$ such that

$$
\Phi_{\lambda}\left(\left(\Gamma_{1}^{-}\right)_{\lambda}\right) \subset M_{\lambda}^{b_{\lambda}^{*}} \text { and } \beta_{\lambda}\left(M_{\lambda}^{b_{\lambda}^{*}}\right) \subset\left(\Gamma_{1}^{+}\right)_{\lambda},
$$

for every $\lambda>\lambda^{*}$, where $M_{\lambda}^{b_{\lambda}^{*}} \doteq I_{\lambda}^{b_{\lambda}^{*}} \cap M_{\lambda}$.

Proof. By Proposition 5.1, there exists $\lambda_{1}>0$ such that $\beta_{\lambda}\left(M_{\lambda}^{b_{\lambda}^{*}}\right) \subset\left(\Gamma_{1}^{+}\right)_{\lambda}$. From Propositions 3.1 and 4.1, we have

$$
\lim _{\lambda \rightarrow \infty}\left(I_{\lambda}\left(\Phi_{\lambda}(y)\right)-b_{\lambda}\right)=0,
$$

independent of $y \in \Gamma_{1 \lambda}^{-}$. Thus, for this $\varepsilon^{*}>0$ there exits $\lambda_{2}=\lambda_{2}\left(\varepsilon^{*}\right)>0$ such that $I_{\lambda}\left(\Phi_{\lambda}(y)\right) \leq b_{\lambda}+\varepsilon^{*}$, for every $\lambda>\lambda_{2}$ and $y \in\left(\Gamma_{1}^{-}\right)_{\lambda}$. Set $\lambda^{*} \doteq \max \left\{\lambda_{1}, \lambda_{2}\right\}$. Hence, $\Phi_{\lambda}\left(\left(\Gamma_{1}^{-}\right)_{\lambda}\right) \subset M_{\lambda}^{b_{\lambda}^{*}}$ and $\beta_{\lambda}\left(M_{\lambda}^{b_{\lambda}^{*}}\right) \subset\left(\Gamma_{1}^{+}\right)_{\lambda}$, for all $\lambda>\lambda^{*}$.

\section{Proof of Theorem 1.1}

We begin by stating a comparison of the topology of the sublevel $M_{\lambda}^{b_{\lambda}^{*}}$ with that of $\Gamma_{1 \lambda}$.

Lemma 6.1. Let $\lambda^{*}>0$ be as in Lemma 5.1. Then

$$
\operatorname{cat}_{M_{\lambda}^{b_{\lambda}^{*}}}\left(M_{\lambda}^{b_{\lambda}^{*}}\right) \geq \operatorname{cat}_{\Gamma_{1 \lambda}}\left(\Gamma_{1 \lambda}\right), \quad \text { for every } \lambda>\lambda^{*} .
$$


Proof. The proof proceeds along the same lines as the proof of $[7$, Lemma 4.3]. Suppose that cat ${ }_{M_{\lambda}^{b_{\lambda}^{*}}}\left(M_{\lambda}^{b_{\lambda}^{*}}\right)=m$. Thus, $M_{\lambda}^{b_{\lambda}^{*}}=\Upsilon_{1} \cup \ldots \cup \Upsilon_{m}$, where $\Upsilon_{j}$ is closed and contractible in $M_{\lambda}^{b_{\lambda}^{*}}$, for $j=1, \ldots, m$. Hence, there exists $h_{j} \in C\left([0,1] \times \Upsilon_{j}, M_{\lambda}^{b_{\lambda}^{*}}\right)$ such that $h_{j}(0, u)=u, h_{j}(1, u)=u_{j} \in M_{\lambda}^{b_{\lambda}^{*}}$ for every $u \in \Upsilon_{j}$ and $j=1, \ldots, m$, for some $u_{j} \in M_{\lambda}^{b_{\lambda}^{*}}$ fixed. Set $B_{j}:=\Phi_{\lambda}^{-1}\left(\Upsilon_{j}\right)$, $j=1, \ldots, m$, which are closed in $\Gamma_{1 \lambda}^{-}$. By Proposition 5.1, we have

$$
\Gamma_{1 \lambda}^{-}=\bigcup_{j=1}^{m} B_{j} .
$$

Using Proposition 5.1 again, the maps $g_{j}:[0,1] \times B_{j} \rightarrow \Gamma_{1 \lambda}^{+}$given by

$$
g_{j}(t, y):=\beta_{\lambda}\left(h_{j}\left(t, \Phi_{\lambda}(y)\right)\right), \quad \text { for all } j \in\{1, \ldots, m\},
$$

are well defined. In addition, $g_{j} \in C\left([0,1] \times B_{j}, \Gamma_{1 \lambda}^{+}\right)$and

$$
g_{j}(0, y)=y, \quad g_{j}(1, y)=y_{j} \in \Gamma_{1 \lambda}^{+}, \quad \text { for every } y \in B_{j}, j=1, \ldots, m,
$$

and $y_{j} \in \Gamma_{1 \lambda}^{+}$fixed, and so $c a t_{\Gamma_{1 \lambda}^{+}} \Gamma_{1 \lambda}^{-} \leq m$. Recalling that $\Gamma_{1 \lambda}^{+}$and $\Gamma_{1 \lambda}^{-}$are homotopically equivalent to $\Gamma_{1 \lambda}$, it follows that $\operatorname{cat}_{\Gamma_{1 \lambda}} \Gamma_{1 \lambda}=\operatorname{cat}_{\Gamma_{1 \lambda}^{+}} \Gamma_{1 \lambda}^{-}$, and hence $\operatorname{cat}_{\Gamma_{1 \lambda}} \Gamma_{1 \lambda} \leq m$, which completes the proof.

Proof of Theorem 1.1. Take $\varepsilon^{*}>0$ given by Proposition 5.1, $\lambda^{*}>0$ given by Proposition 5.1, and suppose $\lambda \geq \lambda^{*}$. If $b_{\lambda}^{*}=b_{\lambda}+\varepsilon$ is a critical value for every $\varepsilon \in\left(0, \varepsilon^{*}\right]$ then $I_{\lambda}$ has infinitely many critical values and the proof is complete. Otherwise, we can assume that $b_{\lambda}^{*}$ is a regular value of $I_{\lambda}$. Since $M_{\lambda}^{b_{\lambda}^{*}}$ is a closed set in $M_{\lambda}$, by Proposition 2.3, the restriction of $I_{\lambda}$ to $M_{\lambda}^{b_{\lambda}^{*}}$ satisfies the $(\mathrm{PS})_{d}$ condition for every $d \in \mathbb{R}$. Hence, by the Lusternik-Schnirelman theory and Lemma 6.1, we obtain $\operatorname{cat}_{\Gamma_{1 \lambda}}\left(\Gamma_{1 \lambda}\right)$ critical points of $\left.I_{\lambda}\right|_{M_{\lambda}^{b_{\lambda}^{*}}}$. By Corollary 2.1, each of these critical points is a critical point of $I_{\lambda}$.

\section{Morse theory for $I_{\lambda}$}

In this section we see how the homology groups of the sets $\Gamma_{1 \lambda},\left(\Gamma_{1}^{-}\right)_{\lambda},\left(\Gamma_{1}^{+}\right)_{\lambda}$ and $M_{\lambda}^{b_{\lambda}^{*}}$ are related. For the convenience of the reader, we repeat the relevant material from [7, Section 5] adapted to our case, thus making the exposition self-contained.

Lemma 7.1. Let $\lambda^{*}>0$ be as in Lemma 5.1. Then

$$
\mathcal{P}_{t}\left(M_{\lambda}^{b_{\lambda}^{*}}\right)=\mathcal{P}_{t}\left(\Gamma_{1 \lambda}\right)+\mathcal{Q}(t),
$$

for every $\lambda \geq \lambda^{*}$, where $\mathcal{Q}$ is a polynomial with non-negative coefficients.

Proof. Setting $\lambda \geq \lambda^{*}$, the function $\Phi_{\lambda}:\left(\Gamma_{1}^{-}\right)_{\lambda} \rightarrow M_{\lambda}$ given by (3.4) induces the homomorphism $\left(\Phi_{\lambda}\right)_{k}: H_{k}\left(\Gamma_{1 \lambda}^{-}\right) \rightarrow H_{k}\left(M_{\lambda}^{b_{\lambda}^{*}}\right)$ between the $k$-th homology groups. Since $\Phi_{\lambda}$ is a injective function, so also is $\left(\Phi_{\lambda}\right)_{k}$. Hence, 
$\operatorname{dim} H_{k}\left(\Gamma_{1 \lambda}^{-}\right) \geq \operatorname{dim} H_{k}\left(M_{\lambda}^{b_{\lambda}^{*}}\right)$, and the result follows from the definition of the Poincaré polynomials and the fact that $\Gamma_{1 \lambda}^{-}$and $\Gamma_{1 \lambda}$ are homotopically equivalent.

Lemma 7.2. Let $\lambda^{*}>0$ be as in Lemma 5.1, $\lambda \geq \lambda^{*}, \delta \in\left(0, \delta_{0}\right)$, for $\delta_{0}$ given by Proposition 2.2, and $b \in(\delta, \infty]$ a noncritical level of $I_{\lambda}$. Then

$$
\mathcal{P}_{t}\left(I_{\lambda}^{b}, I_{\lambda}^{\delta}\right)=t \mathcal{P}_{t}\left(M_{\lambda}^{b}\right) .
$$

Proof. The proof proceeds along the same lines as the proof of [7, Lemma 5.2].

Lemma 7.3. Let $\lambda^{*}, \lambda$ and $\delta$ be as in Lemma 7.2. Then

$$
\mathcal{P}_{t}\left(I_{\lambda}^{b_{\lambda}^{*}}, I_{\lambda}^{\delta}\right)=t \mathcal{P}_{t}\left(\Gamma_{1 \lambda}\right)+t \mathcal{Q}(t)
$$

and

$$
\mathcal{P}_{t}\left(H_{A_{\lambda}}^{1}\left(\Omega_{\lambda}, \Gamma_{0 \lambda}\right), I_{\lambda}^{\delta}\right)=t \mathcal{P}_{t}\left(M_{\lambda}\right)=t,
$$

where $\mathcal{Q}$ is a polynomial with non-negative coefficients.

Proof. As in the proof of Theorem 1.1, we can assume that $b_{\lambda}^{*}$ is a regular value. Applying Lemma 7.2, for $b=b_{\lambda}^{*}$, and Lemma 7.1, we get (7.1). Using that $M_{\lambda}$ is homeomorphic to the unit sphere in $H_{A_{\lambda}}^{1}\left(\Omega_{\lambda}, \Gamma_{0 \lambda}\right)$, which is contractible, we have that $M_{\lambda}$ is contractible. Hence, $\operatorname{dim} H^{k}\left(M_{\lambda}\right)=1$ if $k=0$ and $\operatorname{dim} H^{k}\left(M_{\lambda}\right)=0$ if $k \neq 0$. Finally, (7.2) is obtained by again invoking Lemma 7.2 , for $b=\infty$.

Lemma 7.4. Let $\lambda^{*}, \lambda$ and $\delta$ be as in Lemma 7.2. Then

$$
\mathcal{P}_{t}\left(H_{A_{\lambda}}^{1}\left(\Omega_{\lambda}, \Gamma_{0 \lambda}\right), I_{\lambda}^{b_{\lambda}^{*}}\right)=t^{2}\left[P_{t}\left(\Gamma_{1 \lambda}\right)+\mathcal{Q}(t)-1\right],
$$

where $\mathcal{Q}$ is a polynomial with non-negative coefficients.

Proof. We follow Benci and Cerami [7] in considering the exact sequence:

$$
\begin{aligned}
\cdots \longrightarrow H_{k}\left(H_{A_{\lambda}}^{1}\left(\Omega_{\lambda}, \Gamma_{0 \lambda}\right), I_{\lambda}^{\delta}\right) \stackrel{j_{k}}{\longrightarrow} H_{k}\left(H_{A_{\lambda}}^{1}\left(\Omega_{\lambda}, \Gamma_{0 \lambda}\right), I_{\lambda}^{b_{\lambda}^{*}}\right) \stackrel{\partial_{k}}{\longrightarrow} \\
\quad \stackrel{\partial_{k}}{\longrightarrow} H_{k-1}\left(I_{\lambda}^{b_{\lambda}^{*}}, I_{\lambda}^{\delta}\right) \stackrel{i_{k-1}}{\longrightarrow} H_{k-1}\left(H_{A_{\lambda}}^{1}\left(\Omega_{\lambda}, \Gamma_{0 \lambda}\right), I_{\lambda}^{\delta}\right) \longrightarrow \cdots
\end{aligned}
$$

From (7.2), we obtain $\operatorname{dim} H_{k}\left(H_{A_{\lambda}}^{1}\left(\Omega_{\lambda}, \Gamma_{0 \lambda}\right), I_{\lambda}^{\delta}\right)=0$, for all $k \neq 1$. If we combine this with the fact that the sequence is exact, we see that $\partial_{k}$ is a isomorphism for every $k \geq 3$. Hence,

$$
\operatorname{dim} H_{k}\left(H_{A_{\lambda}}^{1}\left(\Omega_{\lambda}, \Gamma_{0 \lambda}\right), I_{\lambda}^{b_{\lambda}^{*}}\right)=\operatorname{dim} H_{k-1}\left(I_{\lambda}^{b_{\lambda}^{*}}, I_{\lambda}^{\delta}\right), \quad \text { for all } k \geq 3 .
$$

For $k=2$, we have

$$
\begin{aligned}
& \cdots \longrightarrow H_{2}\left(H_{A_{\lambda}}^{1}\left(\Omega_{\lambda}, \Gamma_{0 \lambda}\right), I_{\lambda}^{\delta}\right) \stackrel{j_{2}}{\longrightarrow} H_{2}\left(H_{A_{\lambda}}^{1}\left(\Omega_{\lambda}, \Gamma_{0 \lambda}\right), I_{\lambda}^{b_{\lambda}^{*}}\right) \stackrel{\partial_{2}}{\longrightarrow} \\
& \stackrel{\partial_{2}}{\longrightarrow} H_{1}\left(I_{\lambda}^{b_{\lambda}^{*}}, I_{\lambda}^{\delta}\right) \stackrel{i_{1}}{\longrightarrow} H_{1}\left(H_{A_{\lambda}}^{1}\left(\Omega_{\lambda}, \Gamma_{0 \lambda}\right), I_{\lambda}^{\delta}\right) \longrightarrow \cdots
\end{aligned}
$$


Since $j_{2}$ is sobrejective ( $j_{2}$ is the homomorphism induced by the canonic projection) and $\operatorname{dim} H_{2}\left(H_{A_{\lambda}}^{1}\left(\Omega_{\lambda}, \Gamma_{0 \lambda}\right), I_{\lambda}^{\delta}\right)=0$, by (7.2), we have

$$
H_{2}\left(H_{A_{\lambda}}^{1}\left(\Omega_{\lambda}, \Gamma_{0 \lambda}\right), I_{\lambda}^{b_{\lambda}^{*}}\right)=j_{2}\left(H_{2}\left(H_{A_{\lambda}}^{1}\left(\Omega_{\lambda}, \Gamma_{0 \lambda}\right), I_{\lambda}^{\delta}\right)\right)=\{0\} .
$$

For $k=1$,

$$
\begin{aligned}
\cdots \longrightarrow H_{1}\left(I_{\lambda}^{b_{\lambda}^{*}}, I_{\lambda}^{\delta}\right) \stackrel{i_{1}}{\longrightarrow} & H_{1}\left(H_{A_{\lambda}}^{1}\left(\Omega_{\lambda}, \Gamma_{0 \lambda}\right), I_{\lambda}^{\delta}\right) \stackrel{j_{1}}{\longrightarrow} \\
& \stackrel{j_{1}}{\longrightarrow} H_{1}\left(H_{A_{\lambda}}^{1}\left(\Omega_{\lambda}, \Gamma_{0 \lambda}\right), I_{\lambda}^{b_{\lambda}^{*}}\right) \stackrel{\partial_{1}}{\longrightarrow} H_{0}\left(I_{\lambda}^{b_{\lambda}^{*}}, I_{\lambda}^{\delta}\right) \longrightarrow \cdots
\end{aligned}
$$

Using that $H_{A_{\lambda}}^{1}\left(\Omega_{\lambda}, \Gamma_{0 \lambda}\right)$ is a connected set, we have

$$
H_{0}\left(H_{A_{\lambda}}^{1}\left(\Omega_{\lambda}, \Gamma_{0 \lambda}\right), I_{\lambda}^{b_{\lambda}^{*}}\right)=0 .
$$

We now claim that $i_{1}$ is a isomorphism. Indeed, as $\Gamma_{1 \lambda} \neq \emptyset$ and $\operatorname{dim} H_{0}\left(\Gamma_{1 \lambda}\right)$ is the number of connected components of the set $\Gamma_{1 \lambda}$, we have $H_{0}\left(\Gamma_{1 \lambda}\right) \neq\{0\}$. By $(7.1), H_{1}\left(I_{\lambda}^{b_{\lambda}^{*}}, I_{\lambda}^{\delta}\right) \neq\{0\}$.

From (7.2), we obtain $\operatorname{dim} H_{1}\left(H_{A_{\lambda}}^{1}\left(\Omega_{\lambda}, \Gamma_{0 \lambda}\right), I_{\lambda}^{\delta}\right)=1$. Using that $i_{1}$ is injective, we have $\operatorname{dim} H_{1}\left(I_{\lambda}^{b_{\lambda}^{*}}, I_{\lambda}^{\delta}\right)=1$, and so $i_{1}$ is a isomorphism. Using that $i_{1}$ is a isomorphism and $j_{1}$ is sobrejective, we get

$$
\operatorname{dim} H_{1}\left(H_{A_{A_{\lambda}}}^{1}\left(\Omega_{\lambda}, \Gamma_{0 \lambda}\right), I_{\lambda}^{b_{\lambda}^{*}}\right)=0 .
$$

Combining Lemma 7.3 with (7.4)-(7.7), we have

$$
\begin{aligned}
\mathcal{P}_{t}\left(H_{A_{\lambda}}^{1}\left(\Omega_{\lambda}, \Gamma_{0 \lambda}\right), I_{\lambda}^{b_{\lambda}^{*}}\right) & =\sum_{k \geq 3} t^{k} \operatorname{dim} H_{k}\left(H_{A_{\lambda}}^{1}\left(\Omega_{\lambda}, \Gamma_{0 \lambda}\right), I_{\lambda}^{b_{\lambda}^{*}}\right) \\
& =\sum_{k \geq 3} t^{k} \operatorname{dim} H_{k-1}\left(I_{\lambda}^{b_{\lambda}^{*}}, I_{\lambda}^{\delta}\right)=t \sum_{k \geq 3} t^{k-1} \operatorname{dim} H_{k-1}\left(I_{\lambda}^{b_{\lambda}^{*}}, I_{\lambda}^{\delta}\right) \\
& =t\left[P_{t}\left(I_{\lambda}^{b_{\lambda}^{*}}, I_{\lambda}^{\delta}\right)-t \operatorname{dim} H_{1}\left(I_{\lambda}^{b_{\lambda}^{*}}, I_{\lambda}^{\delta}\right)-\operatorname{dim} H_{0}\left(I_{\lambda}^{b_{\lambda}^{*}}, I_{\lambda}^{\delta}\right)\right] \\
& =t^{2}\left[\mathcal{P}_{t}\left(\Gamma_{1 \lambda}\right)+\mathcal{Q}(t)-1\right] .
\end{aligned}
$$

Lemma 7.5. Let $\lambda^{*}, \lambda$ and $\delta$ be as in Lemma 7.2. Suppose that the set $\mathcal{K}$ of nontrivial solutions of problem (1.5) is discrete. Then

$$
\begin{aligned}
& \sum_{u \in \mathcal{C}_{1}} i_{t}(u)=t \mathcal{P}_{t}\left(\Gamma_{1 \lambda}\right)+t \mathcal{Q}(t)+(1+t) \mathcal{Q}_{1}(t), \\
& \sum_{u \in \mathcal{C}_{2}} i_{t}(u)=t^{2}\left[\mathcal{P}_{t}\left(\Gamma_{1 \lambda}\right)+\mathcal{Q}(t)-1\right]+(1+t) \mathcal{Q}_{2}(t),
\end{aligned}
$$

where $\mathcal{C}_{1} \doteq\left\{u \in \mathcal{K} ; \delta<I_{\lambda}(u) \leq b_{\lambda}^{*}\right\}$ and $\mathcal{C}_{2} \doteq\left\{u \in \mathcal{K} ; b_{\lambda}^{*}<I_{\lambda}(u)\right\}$, and $\mathcal{Q}_{i}$, $i=1,2$, is a polynomial with non-negative coefficients. 
Proof. Using that $I_{\lambda}$ satisfies (PS) condition and applying [6, Theorem I.5.9], there exists a polynomial $\mathcal{Q}_{1}$ with non-negative coefficients such that

$$
\sum_{u \in \mathcal{C}_{1}} i_{t}(u)=\mathcal{P}_{t}\left(I_{\lambda}^{b_{\lambda}^{*}}, I_{\lambda}^{\delta}\right)+(1+t) \mathcal{Q}_{1}(t) .
$$

Hence, (7.8) is a consequence of (7.1) and (7.9) follows from (7.3).

Proof of Theorem 1.2. Let $\lambda^{*}, \lambda$ and $\delta$ be as in Lemma 7.2. Since $I_{\lambda}$ does not have nontrivial solution below the level $\delta_{0}$, we have $\mathcal{K}=\mathcal{C}_{1}+\mathcal{C}_{2}$, for $\mathcal{C}_{1}$ and $\mathcal{C}_{2}$ as in Lemma 7.5. Hence

$$
\sum_{u \in \mathcal{K}} i_{t}(u)=\sum_{u \in \mathcal{C}_{1}} i_{t}(u)+\sum_{u \in \mathcal{C}_{2}} i_{t}(u) .
$$

Using Lemma 7.5, we conclude the proof.

Proof of Corollary 1.1. This is a direct consequence of Theorem 1.2 and the fact that $i_{t}(u)=t^{\mu(u)}$ in the non-degenerate case.

\section{REFERENCES}

[1] L. Abatangelo and S. Terracini, Solutions to nonlinear Schrödinger equations with singular electromagnetic potential and critical exponent, J. Fixed Point Theory Appl. 10 (2011), 147-180.

[2] C.O. Alves, G.M. Figueiredo and M.F. Furtado, Multiple solutions for a nonlinear Schrödinger equation with magnetic fields, J. Differential Equations 251 (2011), 25342548.

[3] A. Ambrosetti, M. Badiale and S. Cingolani, Semiclassical states of nonlinear Schrödinger equations, Arch. Rational Mech. Anal. 140 (1997), 285-300.

[4] A. Ambrosetti And D. Ruiz, Radial solutions concentrating on spheres of nonlinear Schrödinger equations with vanishing potentials, Proc. Royal Soc. Edinburgh Sect. A 136 (2006), 889-907.

[5] S. BARILE, A multiplicity result for singular NLS equations with magnetic potentials, Nonlinear Anal. 68 (2008), 3525-3540.

[6] V. Benci, Introduction to Morse theory. A new approach., Topological Nonlinear Analysis. Progress in Nonlinear Differential Equations and Their Applications, vol. 15 (M. Matzeu et al, eds.), Birkhäuser, Boston, 1995, pp. 37-177.

[7] V. Benci and G. Cerami, Multiple positive solutions of some elliptic problems via the Morse theory and the domain topology, Calc. Var. Partial Differential Equations 2 (1994), 29-48.

[8] The effect of the domain topology on the number of positive solutions of nonlinear elliptic problems, Arch. Ration. Mech. Anal. 114 (1991), 79-93.

[9] H. Berestycki and P.L. Lions, Nonlinear scalar field equations, I, Arch. Ration. Mech. Anal. 82 (1983), 313-346.

[10] H. Brezis, Analyse Fonctionnelle: Théorie et Applications, Collection Mathématiques Appliquées pour la Maîtrise, Masson, Paris, 1983.

[11] A.M. Candela and M. Lazzo, Positive solutions for a mixed boundary problem, Nonlinear Anal. 24 (1995), 1109-1117. 
[12] D. CAO AND Z. TANG, Existence and uniqueness of multi-bump bound states of nonlinear Schrödinger equations with electromagnetic fields, J. Differential Equations 222 (2006), 381-424.

[13] J. Chabrowski And A. Szulkin, On the Schrödinger equation involving a critical Sobolev exponent and magnetic field, Topol. Methods Nonlinear Anal. 25 (2005), 3-21.

[14] S. Cingolani, Semiclassical stationary states of Nonlinear Schrödinger equations with an external magnetic field, J. Differential Equations 188 (2003), 52-79.

[15] S. Cingolanu And M. Clapp, Intertwining semiclassical bound states to a nonlinear magnetic Schrödinger equation, Nonlinearity 22 (2009), 2309-2331.

[16] S. Cingolani, L. Jeanjean and S. Secchi, Multi-peak solutions for magnetic NLS equations without non-degeneracy conditions, ESAIM Control Optim. Calc. Var. 15 (2009), 653-675.

[17] Semiclassical limit for nonlinear Schrödinger equations with electromagnetic fields, J. Math. Anal. Appl. 275 (2002), 108-130.

[18] S. Cingolani And S. SeCCHI, Semiclassical states for NLS equations with magnetic potentials having polynomial growths, J. Math. Phys. 46 (2005), 1-19.

[19] S. Cingolani And G. VAnnella, Multiple positive solutions for a critical quasilinear equation via Morse theory, Ann. Inst. H. Poincaré Anal. Non Linéaire 26 (2009), 397413.

[20] M. Del Pino AND P. Felmer, Local mountain pass for semilinear elliptic problems in unbounded domains, Calc. Var. Partial Differential Equations 4 (1996), 121-137.

[21] I. Ekeland, On the variational principle, J. Math. Anal. Appl. 47 (1974), 324-353.

[22] J.M. Esteban And P.L. Lions, Stationary solutions of nonlinear Schrödinger equations with an external magnetic field, PDE and Calculus of Variations. Progr. Nonlinear Differential Equations Appli., v. 1 (F. Colombini et al., eds.), Birkhäuser, Boston, 1989, pp. 401-449.

[23] L.A. Floer And A. Weinstein, Nonspreading wave packets for the cubic Schrödinger equation with a bounded potential, J. Funct. Anal. 69 (1986), 397-408.

[24] K. KuRATA, Existence and semi-classical limit of the least energy solution to a nonlinear Schrödinger equation with electromagnetic fields, Nonlinear Anal. 62 (2005), 615-628.

[25] G. LI, S. PENG AND C. WANG, Infinitely many solutions for nonlinear Schrödinger equations with electromagnetic fields equation with electromagnetic fields, J. Differential Equations 251 (2011), 3500-3521.

[26] S. LiAng AND J. ZHANG, Solutions of perturbed Schrödinger equations with electromagnetic fields and critical nonlinearity equation with electromagnetic fields, Proc. Edinburgh Math. Soc. 54 (2011), 131-147.

[27] P.H. Rabinowitz, On a class of nonlinear Schrödinger equations, Z. Angew Math. Phys. 43 (1992), 270-291.

[28] M. SquAssina, Soliton dynamics for the nonlinear Schrödinger equation with magnetic field, Manuscripta Math. 130 (2009), 461-494.

[29] W.A. Strauss, Existence of solitary waves in higher dimensions, Comm. Math. Phys. 55 (1977), 149-162.

[30] Z. TANG, Multi-bump bound states of nonlinear Schrödinger equations with electromagnetic fields and critical frequency, J. Differential Equations 245 (2008), 2723-2748.

[31] - On the least energy solutions of nonlinear Schrödinger equations with electromagnetic fields, Computers and Mathematics with Applications 54 (2007), 627-637.

[32] _ Multiplicity of standing wave solutions of nonlinear Schrödinger equations with electromagnetic fields, Z. Angew Math. Phys. 59 (2008), 810-833.

[33] X. WANG AND B. ZENG, On concentration of positive bound states of nonlinear Schrödinger equations with competing potential functions, SIAM J. Math. Anal. 28 (1997), 633-655. 
[34] X. WANG, On concentration of positive bound states of nonlinear Schrödinger equations, Comm. Math. Phys. 153 (1993), 229-244.

[35] Z.-Q. WANG, On the existence of multiple, single-peaked solutions for a semilinear Neumann problem, Arch. Rational Mech. Anal. 120 (1992), 375-399.

[36] M. Willem, Minimax Theorems, Birkhäuser, Boston, 1996.

Manuscript received November 3, 2013

Claudianor O. Alves and Rodrigo C.M. Nemer

Unidade Acadêmica de Matemática e Estatística

Universidade Federal de Campina Grande

58109-970, Campina Grande - PB, BRAZIL

E-mail address: coalves@dme.ufcg.edu.br, rodrigocmnemer@dme.ufcg.edu.br

SÉrgio H.M. SoARES

Departamento de Matemática

Instituto de Ciências Matemáticas e de Computação

Universidade de São Paulo

13560-970, São Carlos - SP, BRAZIL

E-mail address: monari@icmc.usp.br 